\title{
Theory and Applications of Distinctive Conformable Triple Laplace and Sumudu Transforms Decomposition Methods
}

\author{
BHANOTAR Shailesh A. ${ }^{1, *}$ and BELGACEM Fethi Bin Muhammad ${ }^{2}$ \\ ${ }^{1}$ Department of Mathematics, LJIET, L J University, Ahmedabad, Gujarat, India. \\ 2 Department of Mathematics, Faculty of Basic Education, PAAET, Al-Ardiya, Kuwait.
}

Received 20 March 2021; Accepted 5 June 2021

\begin{abstract}
This article presents some important results of conformable fractional partial derivatives. The conformable triple Laplace and Sumudu transform are coupled with the Adomian decomposition method where a new method is proposed to solve nonlinear partial differential equations in 3-space. Moreover, mathematical experiments are provided to verify the performance of the proposed method. A fundamental question that is treated in this work: is whether using the Laplace and Sumudu transforms yield the same results? This question is amply answered in the realm of the proposed applications.
\end{abstract}

AMS Subject Classifications: 35A25, 35M12, 35Q40, 35R11.

Chinese Library Classifications: $\mathrm{O} 211$.

Key Words: Riemann-Liouville fractional integral;fractional derivative; Adomian decomposition method; conformable fractional partial derivative; conformable triple Laplace and Sumudu transform.

\section{Introduction}

In recent years, it has been found that fractional partial differential equations (FPDEs) are very important for modelling many Real-life sciences and engineering applications, such as fluid dynamics, biology of mathematics, electrical circuits, optics, Quantum Mechanics, etc. [1-3]. Many definitions of fractional derivatives and integrals, such as Rizez, Weyl, Riemann-Liouville, Caputo, Hadamard and so on, have been mentioned in literature. These forms of fractional derivatives have many peculiar properties such

*Corresponding author. Email addresses: sbhanotar@gmail.com (Shailesh A. Bhanotar), fbmbelgacem@gmail .com (Fethi Bin Muhammad Belgacem) 
as not all two functions follow chain rule, product, and quotient rule, these significant properties lead to some flaws in physics and engineering applications. In 2014, Khalil et. al [4] introduced a new type of derivative called the conformable fractional derivative (CFD) which satisfies the classical properties of the known derivative. The conformable fractional derivative of function $f:(0, \infty) \rightarrow R$, of order $\alpha \in(0,1]$ is defined by,

$$
T^{\alpha} f(x)=\lim _{h \rightarrow 0} \frac{f\left(x+h x^{1-\alpha}\right)-f(x)}{h} .
$$

Many analytical and numerical methods for accurate and approximate solutions have been developed by several researchers over the last few years, such as the Tanh method [5], Reliable methods [6], Exponential rational function method [7], Kudryashov method [8], Simplest equation method (SEM) [9], Single conformable Laplace transform method (CLT) [10], single conformable Laplace transform method (CLT) [10], conformable double Laplace transform (CDLT) [11,12], conformable triple Laplace transform and Sumudu transform [34,35], double Shehu transform [13], and some critical analyses about conformable fractional [20].

Watugala [14], implemented a new integral transformation at the beginning of the 1990s, called the Sumudu transformation (ST), which derived from the classical Fourier Transform, and applied it to obtain the solution of many problems in real life science and engineering. Infect, it is proved to be an efficient method for solving physical problems because of its unit and scale preserving properties. For more about (ST) see $[15,16]$, authors have studied about properties of Sumudu transform and $[17,18]$ application of Sumudu transform (ST) and Laplace transform (LT).

The transformation of Sumudu is defined in the set of functions [33],

$$
\mathcal{A}=\left\{f(t)\left|\exists K, \tau_{1}, \tau_{2}>0,\right| f(t) \mid<K e^{\frac{|t|}{\tau_{j}}}, \text { if } t \epsilon(-1)^{j} \times[0, \infty)\right\}
$$

By the following formula,

$$
F(u)=S[f(t)]=\int_{0}^{\infty} f(u t) e^{-t} \mathrm{~d} u, \quad u \in\left(-\tau_{1}, \tau_{2}\right) .
$$

The authors in $[19,21]$ recently introduced the single and double conformable Sumudu transform (CST) in 2019-20. Ibrahim et.al [36-38], have explored operator for symmetric conformable fractional derivative of complex variable and on quantum hybrid fractional conformable differential in a complex domain, in [39,40], on subclasses of analytic functions based on a quantum symmetric and the generalized wave dynamical equations based on time space symmetric differential equation operator, respectively, and Moreover, in [41], some fixed-point theorems for almost weak contraction in S- metric space via conformable fractional operator. In order to solve linear fractional partial differential equations in the conformable fractional derivative sense, we implement the conformable Triple Sumudu transform (CDST) due to the certain benefits of Sumudu transformation 
(ST) over Laplace Transformation (LT). After referring literature, we come up with the idea to study the nonlinear partial fractional differential equations by defining a function in three-dimensional space. Therefore, a Conformable triple Sumudu transform is defined and coupled with Adomian decomposition method to solve systematic nonlinear partial fractional differential equations. The main advantage of this method will give accurate solutions to nonlinear partial fractional derivatives in three dimensional space. One thing you will be able to get the answer of question asked by Atangana et al. [31], in his paper.

This paper is divided into the following sections: In Section 2, some basic Fractional calculus concept has been discussed on conformable partial derivatives. In Sections 3 and 4, the main results and theorems on the conformable triple Sumudu and Laplace transforms are investigated respectively. In Section 5, Sumudu and Laplace transform Adomian decomposition methods for a general nonhomogeneous partial fractional differential equation are given. In Section 6, numerical experiment is conducted using the proposed method to validate the obtained results, analyzed and presented plots for different parameters. In Section 7, a conclusion of our research work is provided.

\section{Preliminary}

The basic definitions and properties of the fractional calculus theory are provided in this section, which are further used in this paper.

Definition 2.1. The Riemann-Liouville fractional integral operator of order $\gamma>0$, for $t>0$ is defined by

$$
J^{\gamma} f(t)=\frac{1}{\Gamma(\gamma)} \int_{0}^{t}(t-\varepsilon)^{\gamma-1} f(\varepsilon) \mathrm{d} \varepsilon \quad \text { and } J^{0} f(t)=f(t) .
$$

And the Riemann-Liouville derivative of order $\gamma>0$ is given by

$$
D_{t}^{\gamma} f(t)=\frac{\mathrm{d}^{n}}{\mathrm{~d} t^{n}}\left(J^{n-\gamma} f(t)\right)=\frac{\mathrm{d}^{n}}{\mathrm{~d} t^{n}}\left(\frac{1}{\Gamma(n-\gamma)} \int_{0}^{t}(t-u)^{n-\gamma-1} f(u) \mathrm{d} u\right),
$$

where $n-1<\gamma<n$, (n a positive integer) and then take nth-order derivative.

When attempting to model real-world phenomena with fractional differential equations, the Riemann-Liouville derivative has a certain drawback. We shall therefore implement an updated fractional differential operator suggested by M. Caputo in his dissertation on viscoelasticity theory [25].

The properties of the operator $J^{\gamma}$ can be found, where the gamma function is $\Gamma(\gamma)$, $\gamma>0$ and $t>0$.

Definition 2.2. The Caputo fractional derivative of $f(t)$ of order $\gamma>0$ with $t>0$ is defined as,

$$
J^{m-\gamma} D^{m} f(t)=\frac{1}{\Gamma(m-\gamma)} \int_{0}^{t}(t-\varepsilon)^{m-\gamma-1} f^{(m)}(\varepsilon) \mathrm{d} \varepsilon,
$$


for $m-1<\gamma \leq m, m \in N$.

Definition 2.3. (see [32]). Given a function $f: R^{+} \times R^{+} \times R^{+} \rightarrow R$, the conformable partial fractional derivatives (CPFDs) of order $\alpha, \beta$ and $\gamma$ of the function $f\left(\frac{x^{\alpha}}{\alpha}, \frac{y^{\beta}}{\beta}, \frac{t^{\gamma}}{\gamma}\right)$ is defined as follows:

$$
\begin{aligned}
& \partial_{x}^{\alpha} f=\frac{\partial^{\alpha}}{\partial x^{\alpha}} f\left(\frac{x^{\alpha}}{\alpha}, \frac{y^{\beta}}{\beta}, \frac{t^{\gamma}}{\gamma}\right)=\lim _{h \rightarrow 0} \frac{f\left(\frac{x^{\alpha}}{\alpha}+h x^{1-\alpha}, \frac{y^{\beta}}{\beta}, \frac{t^{\gamma}}{\gamma}\right)-f\left(\frac{x^{\alpha}}{\alpha}, \frac{y^{\beta}}{\beta}, \frac{t^{\gamma}}{\gamma}\right)}{h}, \\
& \partial_{y}^{\beta} f=\frac{\partial^{\beta}}{\partial y^{\beta}} f\left(\frac{x^{\alpha}}{\alpha}, \frac{y^{\beta}}{\beta}, \frac{t^{\gamma}}{\gamma}\right)=\lim _{k \rightarrow 0} \frac{f\left(\frac{x^{\alpha}}{\alpha}, \frac{y^{\beta}}{\beta}+k y^{1-\beta}, \frac{t^{\gamma}}{\gamma}\right)-f\left(\frac{x^{\alpha}}{\alpha}, \frac{y^{\beta}}{\beta}, \frac{t^{\gamma}}{\gamma}\right)}{k}, \\
& \partial_{t}^{\gamma} f=\frac{\partial^{\gamma}}{\partial t^{\gamma}} f\left(\frac{x^{\alpha}}{\alpha}, \frac{y^{\beta}}{\beta}, \frac{t^{\gamma}}{\gamma}\right)=\lim _{\varepsilon \rightarrow 0} \frac{f\left(\frac{x^{\alpha}}{\alpha}, \frac{y^{\beta}}{\beta}, \frac{t^{\gamma}}{\gamma}+\epsilon t^{1-\gamma}\right)-f\left(\frac{x^{\alpha}}{\alpha}, \frac{y^{\beta}}{\beta}, \frac{t^{\gamma}}{\gamma}\right)}{\epsilon},
\end{aligned}
$$

where $0<\alpha, \beta, \gamma \leq 1, \frac{x^{\alpha}}{\alpha}, \frac{y^{\beta}}{\beta}, \frac{t^{\gamma}}{\gamma}>0, \partial_{x}^{\alpha}=\frac{\partial^{\alpha}}{\partial x^{\alpha}}, \partial_{y}^{\beta}=\frac{\partial^{\beta}}{\partial y^{\beta}}$ and $\partial_{t}^{\gamma}=\frac{\partial^{\gamma}}{\partial t^{\gamma}}$ are called the fractional partial derivatives of order $\alpha, \beta$, and $\gamma$, respectively.

We prove the basic Theorem 2.1, and the relation between the CPFDs and partial derivatives as follows:

Theorem 2.1. Let $\alpha, \beta, \gamma \in(0,1]$ and $f(x, y, t)$ be a differentiable at a point for $x, y, t>0$. Then,

1. $\partial_{x}^{\alpha} f=\frac{\partial^{\alpha}}{\partial x^{a}} f(x, y, t)=x^{1-\alpha} \frac{\partial f(x, y, t)}{\partial x}=x^{1-\alpha} \partial_{x} f$.

2. $\partial_{y}^{\beta} f=\frac{\partial^{\beta}}{\partial y^{\beta}} f(x, y, t)=y^{1-\beta} \frac{\partial f(x, y, t)}{\partial y}=y^{1-\beta} \partial_{y} f$.

3. $\partial_{t}^{\gamma} f=\frac{\partial^{\gamma}}{\partial t \gamma} f(x, y, t)=t^{1-\gamma} \frac{\partial f(x, y, t)}{\partial t}=t^{1-\gamma} \partial_{t} f$.

Proof. By the definition of CFPD,

$$
\begin{aligned}
\frac{\partial^{\alpha}}{\partial x^{\alpha}} f(x, y, t) & =\lim _{h \rightarrow 0} \frac{f\left(x+h x^{1-\alpha}, y, t\right)-f(x, y, t)}{h}, \quad \text { taking } h x^{1-\alpha}=\varphi \\
& =\lim _{\varphi \rightarrow 0} \frac{f(x+\varphi, y, t)-f(x, y, t)}{\varphi x^{\alpha-1}} \\
& =x^{1-\alpha} \lim _{\varphi \rightarrow 0} \frac{f(x+\varphi, y, t)-f(x, y, t)}{\varphi} \\
& =x^{1-\alpha} \frac{\partial f(x, y, t)}{\partial x} .
\end{aligned}
$$

Similarity, we can prove the results (2) and (3). 
In the next proposition, we mention the conformable partial fractional derivative of some functions. By using Theorem 2.1, it can be verified easily.

Proposition 2.1. Let $\alpha, \beta, \gamma \in(0,1]$ and $a, b \in R, l, m, n \in N$; Then, we have the followings:

1. $\frac{\partial^{\alpha}}{\partial x^{\alpha}}\left(a u\left(\frac{x^{\alpha}}{\alpha}, \frac{y^{\beta}}{\beta}, \frac{t^{\gamma}}{\gamma}\right)+b v\left(\frac{x^{\alpha}}{\alpha}, \frac{y^{\beta}}{\beta}, \frac{t^{\gamma}}{\gamma}\right)\right)=a \frac{\partial^{\alpha}}{\partial x^{\alpha}} u\left(\frac{x^{\alpha}}{\alpha}, \frac{y^{\beta}}{\beta}, \frac{t^{\gamma}}{\gamma}\right)+b \frac{\partial^{\alpha}}{\partial x^{\alpha}} v\left(\frac{x^{\alpha}}{\alpha}, \frac{y^{\beta}}{\beta}, \frac{t^{\gamma}}{\gamma}\right)$.

2. $\frac{\partial^{\alpha+\beta+\gamma}}{\partial x^{\alpha} \partial y^{\beta} \partial t \gamma}\left(\left(\frac{x^{\alpha}}{\alpha}\right)^{l}\left(\frac{y^{\beta}}{\beta}\right)^{m}\left(\frac{t^{\gamma}}{\gamma}\right)^{n}\right)=\operatorname{lmn}\left(\frac{x^{\alpha}}{\alpha}\right)^{l-\alpha}\left(\frac{y^{\beta}}{\beta}\right)^{m-\beta}\left(\frac{t^{\gamma}}{\gamma}\right)^{n-\gamma}$.

3. $\frac{\partial^{\alpha}}{\partial x^{\alpha}}\left(\left(\frac{x^{\alpha}}{\alpha}\right)^{l}\left(\frac{t^{\gamma}}{\gamma}\right)^{n}\right)=l\left(\frac{x^{\alpha}}{\alpha}\right)^{l-1}\left(\frac{t^{\gamma}}{\gamma}\right)^{n}$ and

$\frac{\partial^{\gamma}}{\partial t^{\gamma}}\left(\left(\frac{x^{\alpha}}{\alpha}\right)^{l}\left(\frac{y^{\beta}}{\beta}\right)^{m}\left(\frac{t^{\gamma}}{\gamma}\right)^{n}\right)=n\left(\frac{x^{\alpha}}{\alpha}\right)^{l}\left(\frac{y^{\beta}}{\beta}\right)^{m}\left(\frac{t^{\gamma}}{\gamma}\right)^{n-1}$.

4. $\frac{\partial^{\alpha}}{\partial x^{\alpha}}\left(\sin \left(\frac{x^{\alpha}}{\alpha}\right) \cos \left(\frac{t^{\gamma}}{\gamma}\right)\right)=\cos \left(\frac{x^{\alpha}}{\alpha}\right) \cos \left(\frac{t^{\gamma}}{\gamma}\right)$.

5. $\frac{\partial^{\beta}}{\partial y^{\beta}}\left(\sin \left(\frac{x^{\alpha}}{\alpha}\right) \cos \left(\frac{y^{\beta}}{\beta}\right) \cos \left(\frac{t^{\gamma}}{\gamma}\right)\right)=-\sin \left(\frac{x^{\alpha}}{\alpha}\right) \sin \left(\frac{y^{\beta}}{\beta}\right) \cos \left(\frac{t^{\gamma}}{\gamma}\right)$.

Proof. It can be easily verified with the help of Theorem 2.1.

\section{Some results and theorems of the conformable fractional triple Laplace transform}

In this section, we recall some basic definitions on fractional conformable Laplace transform and some results which will be used later on. Also, we define conformable triple Laplace transform. For more details see [25-30].

Definition 3.1. Let the function $u:(0, \infty) \rightarrow R$ and $0<\alpha \leq 1$ be the piecewise continuous function. Then, the conformable Laplace Transform (CLT) of function $u\left(\frac{x^{\alpha}}{\alpha}\right)$ of exponential of order, is defined and denoted by,

$$
U_{\alpha}(p)=L_{x}^{\alpha}\left(u\left(\frac{x^{\alpha}}{\alpha}\right)\right)=\int_{0}^{\infty} e^{-p\left(\frac{x^{\alpha}}{\alpha}\right)} u\left(\frac{x^{\alpha}}{\alpha}\right) x^{\alpha-1} \mathrm{~d} x, \quad x>0 .
$$

Definition 3.2. Let $u(x, y)$ be a piece wise continuous function on the domain $D$ of $R^{+} \times R^{+}$of exponential order. Then Fractional conformable double Laplace Transform (FCDLT) of $u\left(\frac{x^{\alpha}}{\alpha}, \frac{y^{\beta}}{\beta}\right)$ is defined and denoted by,

$$
L_{x}^{\alpha} L_{y}^{\beta}\left(u\left(\frac{x^{\alpha}}{\alpha}, \frac{y^{\beta}}{\beta}\right)\right)=U_{\alpha, \beta}(p, q)=\int_{0}^{\infty} \int_{0}^{\infty} e^{-p\left(\frac{x^{\alpha}}{\alpha}\right)-q\left(\frac{y^{\beta}}{\beta}\right)} u\left(\frac{x^{\alpha}}{\alpha}, \frac{y^{\beta}}{\beta}\right) x^{\alpha-1} y^{\beta-1} \mathrm{~d} x \mathrm{~d} y,
$$


where $\frac{x^{\alpha}}{\alpha}, \frac{y^{\beta}}{\beta}>0, p, q \in \mathbb{C}, \alpha, \beta \in(0,1]$.

Now, we define fractional conformable triple Laplace transform, for $\alpha, \beta, \gamma \in(0,1]$, and $p, q \in \mathbb{C}$ are the Laplace variables.

Definition 3.3. Let $u\left(\frac{x^{\alpha}}{\alpha}, \frac{y^{\beta}}{\beta}, \frac{t^{\gamma}}{\gamma}\right)$ be a real valued piecewise continuous function of $x, y$ and $t$ defined on the domain $\mathrm{D}$ of $R^{+} \times R^{+} \times R^{+}$of exponential order. Then, the fractional conformable triple Laplace transform (FCTLT) of $u\left(\frac{x^{\alpha}}{\alpha}, \frac{y^{\beta}}{\beta}, \frac{t^{\gamma}}{\gamma}\right)$ is defined as follows:

$$
\begin{aligned}
& L_{x}^{\alpha} L_{y}^{\beta} L_{t}^{\gamma}\left(u\left(\frac{x^{\alpha}}{\alpha}, \frac{y^{\beta}}{\beta}, \frac{t^{\gamma}}{\gamma}\right)\right)=U_{\alpha, \beta, \gamma}(p, q, s) \\
= & \int_{0}^{\infty} \int_{0}^{\infty} \int_{0}^{\infty} e^{-p\left(\frac{x^{\alpha}}{\alpha}\right)-q\left(\frac{y^{\beta}}{\beta}\right)-s\left(\frac{t^{\gamma}}{\gamma}\right)} u\left(\frac{x^{a}}{\alpha}, \frac{y^{\beta}}{\beta}, \frac{t^{\gamma}}{\gamma}\right) x^{\alpha-1} y^{\beta-1} t^{\gamma-1} \mathrm{~d} x \mathrm{~d} y \mathrm{~d} t,
\end{aligned}
$$

where $p, q, s \in \mathbb{C}$, are Laplace variables of $\frac{x^{\alpha}}{\alpha}, \frac{y^{\beta}}{\beta}$ and $\frac{t^{\gamma}}{\gamma}$ respectively and $\alpha, \beta, \gamma \in(0,1]$.

The fractional conformable inverse triple Laplace transform, denoted by $u\left(\frac{x^{\alpha}}{\alpha}, \frac{y^{\beta}}{\beta}, \frac{t \gamma}{\gamma}\right)$ is defined by,

$$
\begin{aligned}
& u\left(\frac{x^{\alpha}}{\alpha}, \frac{y^{\beta}}{\beta}, \frac{t^{\gamma}}{\gamma}\right)=L_{p}^{-1} L_{q}^{-1} L_{s}^{-1}\left(U_{\alpha, \beta, y}(p, q, s)\right) \\
= & \frac{1}{2 \pi i} \int_{\alpha-i \infty}^{\alpha+i \infty} e^{p\left(\frac{x^{a}}{a}\right)}\left[\frac{1}{2 \pi i} \int_{\beta-i \infty}^{\beta+i \infty} e^{q\left(\frac{y^{\beta}}{\beta}\right)}\left[\frac{1}{2 \pi i} \int_{\gamma-i \infty}^{\gamma+i \infty} e^{s\left(\frac{t \gamma}{\gamma}\right)} U_{\alpha, \beta, \gamma}(p, q, s) \mathrm{d} s\right] \mathrm{d} q\right] \mathrm{d} p .
\end{aligned}
$$

Definition 3.4 A unit step or Heaviside unit step function is defined as follows:

$$
H\left(\left(\frac{x^{\alpha}}{\alpha}\right)-a,\left(\frac{y^{\beta}}{\beta}\right)-b,\left(\frac{t^{\gamma}}{\gamma}\right)-c\right)= \begin{cases}1 ; & \frac{x^{\alpha}}{\alpha}>a, \frac{y^{\beta}}{\beta}>b, \frac{t^{\gamma}}{\gamma}>c \\ 0 ; & \frac{x^{\alpha}}{\alpha}<a, \frac{y^{\beta}}{\beta}<b, \frac{t^{\gamma}}{\gamma}<c .\end{cases}
$$

Theorem 3.1. If $L_{x}^{\alpha} L_{y}^{\beta} L_{t}^{\gamma}\left(u\left(\frac{x^{\alpha}}{\alpha}, \frac{y^{\beta}}{\beta}, \frac{t^{\gamma}}{\gamma}\right)\right)=U_{\alpha, \beta, \gamma}(p, q, s)$, and $L_{x}^{\alpha} L_{y}^{\beta} L_{t}^{\gamma}\left(v\left(\frac{x^{\alpha}}{\alpha}, \frac{y^{\beta}}{\beta}, \frac{t^{\gamma}}{\gamma}\right)\right)$ $=V_{\alpha, \beta, \gamma}(p, q, s)$ and $\mathrm{A}, \mathrm{B}$ and $\mathrm{C}$ are constants then the followings hold:

1. Linearity property:

$$
\begin{aligned}
& L_{x}^{\alpha} L_{y}^{\beta} L_{t}^{\gamma}\left(A u\left(\frac{x^{\alpha}}{\alpha}, \frac{y^{\beta}}{\beta}, \frac{t^{\gamma}}{\gamma}\right)+B v\left(\frac{x^{\alpha}}{\alpha}, \frac{y^{\beta}}{\beta}, \frac{t^{\gamma}}{\gamma}\right)\right) \\
& =A L_{x}^{\alpha} L_{y}^{\beta} L_{t}^{\gamma}\left(u\left(\frac{x^{\alpha}}{\alpha}, \frac{y^{\beta}}{\beta}, \frac{t^{\gamma}}{\gamma}\right)\right)+B L_{x}^{\alpha} L_{y}^{\beta} L_{t}^{\gamma}\left(v\left(\frac{x^{\alpha}}{\alpha}, \frac{y^{\beta}}{\beta}, \frac{t^{\gamma}}{\gamma}\right)\right) \\
& =A U_{\alpha, \beta, \gamma}(p, q, s)+B V_{\alpha, \beta, \gamma}(p, q, s) .
\end{aligned}
$$

2. $L_{x}^{\alpha} L_{y}^{\beta} L_{t}^{\gamma}(C)=\frac{C}{p q s^{\prime}}$ where $C$ is the constant. 
3. $L_{x}^{\alpha} L_{y}^{\beta} L_{t}^{\gamma}\left(\left(\frac{x^{\alpha}}{\alpha}\right)^{l}\left(\frac{y^{\beta}}{\beta}\right)^{m}\left(\frac{t^{\gamma}}{\gamma}\right)^{n}\right)=\frac{\Gamma(l+1) \Gamma(m+1) \Gamma(n+1)}{p^{l+1} q^{m+1} s^{n+1}}$,

where $\Gamma(\cdot)$ Is the gamma function. Note that $\Gamma(n+1)=n !$, for $\mathrm{n}=0,1,2,3, \ldots$

4. The First Shifting theorem for conformable triple Laplace transform:

If $L_{x}^{\alpha} L_{y}^{\beta} L_{t}^{\gamma}\left(u\left(\frac{x^{\alpha}}{\alpha}, \frac{y^{\beta}}{\beta}, \frac{t^{\gamma}}{\gamma}\right)\right)=U_{\alpha, \beta, \gamma}(p, q, s)$, then $L_{x}^{\alpha} L_{y}^{\beta} L_{t}^{\gamma}\left(e^{a\left(\frac{x^{\alpha}}{\alpha}\right)+b\left(\frac{y^{\beta}}{\beta}\right)+c\left(\frac{t \gamma}{\gamma}\right)} u\left(\frac{x^{\alpha}}{\alpha}, \frac{y^{\beta}}{\beta}, \frac{t^{\gamma}}{\gamma}\right)\right)=U_{\alpha, \beta, \gamma}(p-a, q-b, s-c)$.

5. If $L_{x}^{\alpha} L_{y}^{\beta} L_{t}^{\gamma}\left(u\left(\frac{x^{\alpha}}{\alpha}, \frac{y^{\beta}}{\beta}, \frac{t^{\gamma}}{\gamma}\right)\right)=U_{\alpha, \beta, \gamma}(p, q, s)$, then $L_{x}^{\alpha} L_{y}^{\beta} L_{t}^{\gamma}\left(\left(\frac{x^{\alpha}}{\alpha}\right)^{l}\left(\frac{y^{\beta}}{\beta}\right)^{m}\left(\frac{t^{\gamma}}{\gamma}\right)^{n} u\left(\frac{x^{\alpha}}{\alpha}, \frac{y^{\beta}}{\beta}, \frac{t^{\gamma}}{\gamma}\right)\right)=(-1)^{l+m+n} \frac{\mathrm{d}^{l+m+n}}{\mathrm{~d} p^{l} \mathrm{~d} q^{m} \mathrm{~d} s^{n}}\left(U_{\alpha, \beta, \gamma}(p, q, s)\right)$.

6. $L_{x}^{\alpha} L_{y}^{\beta} L_{t}^{\gamma}\left(\sin \left(A \frac{x^{\alpha}}{\alpha}\right) \sin \left(B \frac{y^{\beta}}{\beta}\right) \sin \left(C \frac{t^{\gamma}}{\gamma}\right)\right)=\frac{A B C}{\left(p^{2}+A^{2}\right)\left(q^{2}+B^{2}\right)\left(s^{2}+C^{2}\right)}$ and $L_{x}^{\alpha} L_{y}^{\beta} L_{t}^{\gamma}\left(\cos \left(A \frac{x^{\alpha}}{\alpha}\right) \cos \left(B \frac{y^{\beta}}{\beta}\right) \cos \left(C \frac{t^{\gamma}}{\gamma}\right)\right)=\frac{p q s}{\left(p^{2}+A^{2}\right)\left(q^{2}+B^{2}\right)\left(s^{2}+C^{2}\right)}$.

Proof. It can be proved using definition of Triple Laplace transform.

Theorem 3.2. If $L_{x}^{\alpha} L_{y}^{\beta} L_{t}^{\gamma}\left(u\left(\frac{x^{\alpha}}{\alpha}, \frac{y^{\beta}}{\beta}, \frac{t^{\gamma}}{\gamma}\right)\right)=U_{\alpha, \beta, \gamma}(p, q, s)$, then

$$
L_{x}^{\alpha} L_{y}^{\beta} L_{t}^{\gamma}\left(\begin{array}{c}
u\left(\frac{x^{\alpha}}{\alpha}-\frac{\zeta^{\alpha}}{\alpha}, \frac{y^{\beta}}{\beta}-\frac{\eta^{\beta}}{\beta}, \frac{t^{\gamma}}{\gamma}-\frac{\theta^{\gamma}}{\gamma}\right) \\
H\left(\frac{x^{\alpha}}{\alpha}-\frac{\zeta^{\alpha}}{\alpha}, \frac{y^{\beta}}{\beta}-\frac{\eta^{\beta}}{\beta}, \frac{t^{\gamma}}{\gamma}-\frac{\theta^{\gamma}}{\gamma}\right)
\end{array}\right)=e^{-p\left(\frac{\zeta^{\alpha}}{\alpha}\right)-q\left(\frac{\eta^{\beta}}{\beta}\right)-s\left(\frac{\theta^{\gamma}}{\gamma}\right)} U_{\alpha, \beta, \gamma}(p, q, s),
$$

where $H(x, y, t)$ is a Heaviside unit step function as defined in eq. (3.3).

Proof. By applying the definition of CTLT,

$$
\begin{aligned}
\text { LHS } & =L_{x}^{\alpha} L_{y}^{\beta} L_{t}^{\gamma}\left(u\left(\frac{x^{\alpha}}{\alpha}-\frac{\zeta^{\alpha}}{\alpha}, \frac{y^{\beta}}{\beta}-\frac{\eta^{\beta}}{\beta}, \frac{t^{\gamma}}{\gamma}-\frac{\theta^{\gamma}}{\gamma}\right) H\left(\frac{x^{\alpha}}{\alpha}-\frac{\zeta^{\alpha}}{\alpha}, \frac{y^{\beta}}{\beta}-\frac{\eta^{\beta}}{\beta}, \frac{t^{\gamma}}{\gamma}-\frac{\theta^{\gamma}}{\gamma}\right)\right) \\
& =\int_{0}^{\infty} \int_{0}^{\infty} \int_{0}^{\infty}\left(\begin{array}{c}
e^{-p\left(\frac{x^{\alpha}}{\alpha}\right)-q\left(\frac{y^{\beta}}{\beta}\right)-s\left(\frac{t^{r}}{\gamma}\right)} u\left(\frac{x^{\alpha}}{a}-\frac{\zeta^{\alpha}}{\alpha}, \frac{y^{\beta}}{\beta}-\frac{\eta^{\beta}}{\beta}, \frac{t^{\gamma}}{\gamma}-\frac{\theta^{\gamma}}{\gamma}\right) \\
H\left(\frac{x^{\alpha}}{\alpha}-\frac{\zeta^{\alpha}}{\alpha}, \frac{y^{\beta}}{\beta}-\frac{\eta^{\beta}}{\beta}, \frac{t^{\gamma}}{\gamma}-\frac{\theta^{\gamma}}{\gamma}\right) x^{\alpha-1} y^{1-\beta} t^{1-\gamma}
\end{array}\right) \mathrm{d} x \mathrm{~d} y \mathrm{~d} t .
\end{aligned}
$$

Now, using definition of Heaviside Unit step function $H(x, y, t)$, we have

$$
=\int_{\frac{\zeta^{\alpha}}{\alpha}}^{\infty} \int_{\frac{\eta^{\beta}}{\beta}}^{\infty} \int_{\frac{\theta^{\gamma}}{\gamma}}^{\infty}\left(\begin{array}{c}
e^{-p\left(\frac{x^{\alpha}}{\alpha}\right)-q\left(\frac{y^{\beta}}{\beta}\right)-s\left(\frac{t^{\gamma}}{\gamma}\right)} \\
u\left(\frac{x^{\alpha}}{\alpha}-\frac{\zeta^{\alpha}}{\alpha}, \frac{y^{\beta}}{\beta}-\frac{\eta^{\beta}}{\beta}, \frac{t^{\gamma}}{\gamma}-\frac{\theta^{\gamma}}{\gamma}\right)
\end{array}\right) \quad x^{\alpha-1} y^{\beta-1} t^{\gamma-1} \mathrm{~d} x \mathrm{~d} y \mathrm{~d} t .
$$


By putting $\frac{x^{\alpha}}{\alpha}-\frac{\zeta^{\alpha}}{\alpha}=\frac{\mathcal{A}^{\alpha}}{\alpha}, \frac{y^{\beta}}{\beta}-\frac{\eta^{\beta}}{\beta}=\frac{\tau^{\beta}}{\beta}, \frac{t^{\gamma}}{\gamma}-\frac{\theta^{\gamma}}{\gamma}=\frac{u^{\gamma}}{\gamma}$, we have,

$$
=e^{-p\left(\frac{\tau^{\alpha}}{\alpha}\right)-q\left(\frac{\eta^{\beta}}{\beta}\right)-s\left(\frac{\theta^{\gamma}}{\gamma}\right)} \int_{0}^{\infty} \int_{0}^{\infty} \int_{0}^{\infty}\left(\begin{array}{c}
e^{-p\left(\frac{\mathcal{A}^{\alpha}}{\alpha}\right)-q\left(\frac{\mathcal{T} \beta}{\beta}\right)-s\left(\frac{\mathcal{U}^{\gamma}}{\gamma}\right)} \\
u\left(\frac{\mathcal{A}^{\alpha}}{\alpha}, \frac{\mathcal{T}^{\beta}}{\beta}, \frac{\mathcal{U}^{\gamma}}{\gamma}\right)
\end{array}\right) \mathcal{A}^{\alpha-1} \mathcal{T}^{\beta-1} \mathcal{U}^{\gamma-1} \mathrm{~d} \mathcal{A} \mathrm{d} \mathcal{T} \mathrm{d} \mathcal{U},
$$

which gives,

$$
\begin{aligned}
& L_{x}^{\alpha} L_{y}^{\beta} L_{t}^{\gamma}\left(u\left(\frac{x^{\alpha}}{\alpha}-\frac{\zeta^{\alpha}}{\alpha}, \frac{y^{\beta}}{\beta}-\frac{\eta^{\beta}}{\beta}, \frac{t^{\gamma}}{\gamma}-\frac{\theta^{\gamma}}{\gamma}\right) H\left(\frac{x^{\alpha}}{\alpha}-\frac{\zeta^{\alpha}}{\alpha}, \frac{y^{\beta}}{\beta}-\frac{\eta^{\beta}}{\beta}, \frac{t^{\gamma}}{\gamma}-\frac{\theta^{\gamma}}{\gamma}\right)\right) \\
= & e^{-p\left(\frac{\zeta^{\alpha}}{\alpha}\right)-q\left(\frac{\eta^{\beta}}{\beta}\right)-s\left(\frac{\theta^{\gamma}}{\gamma}\right)} U_{\alpha, \beta, \gamma}(p, q, s) .
\end{aligned}
$$

This completes the proof of the theorem.

Theorem 3.3. If $L_{x}^{\alpha} L_{y}^{\beta} L_{t}^{\gamma}\left(u\left(\frac{x^{\alpha}}{\alpha}, \frac{y^{\beta}}{\beta}, \frac{t^{\gamma}}{\gamma}\right)\right)=U_{\alpha, \beta, \gamma}(p, q, s)$. Then the fractional conformable triple Laplace transform of the function $\left(\frac{x^{\alpha}}{\alpha}\right)^{l} u(x, y, t),\left(\frac{y^{\beta}}{\beta}\right)^{m} u\left(\frac{x^{\alpha}}{\alpha}, \frac{y^{\beta}}{\beta}, \frac{t^{\gamma}}{\gamma}\right),\left(\frac{t^{\gamma}}{\gamma}\right)^{n} u\left(\frac{x^{\alpha}}{\alpha}, \frac{y^{\beta}}{\beta}, \frac{t \gamma}{\gamma}\right)$ and $\left(\frac{x^{\alpha}}{\alpha}\right)^{l} \frac{\partial^{\beta+\gamma}}{\partial y^{\beta} \partial t \gamma}(u(x, y, t))$ are given $b y$,

1. $L_{x}^{\alpha} L_{y}^{\beta} L_{t}^{\gamma}\left(\left(\frac{x^{\alpha}}{\alpha}\right)^{l} u\left(\frac{x^{\alpha}}{\alpha}, \frac{y^{\beta}}{\beta}, \frac{t^{\gamma}}{\gamma}\right)\right)=(-1)^{l} \frac{\mathrm{d}^{l}}{\mathrm{~d} p^{l}}\left(U_{\alpha, \beta, \gamma}(p, q, s)\right)$.

2. $L_{x}^{\alpha} L_{y}^{\beta} L_{t}^{\gamma}\left(\left(\frac{y^{\beta}}{\beta}\right)^{m} u\left(\frac{x^{\alpha}}{\alpha}, \frac{y^{\beta}}{\beta}, \frac{t^{\gamma}}{\gamma}\right)\right)=(-1)^{m} \frac{\mathrm{d}^{m}}{\mathrm{~d} q^{m}}\left(U_{\alpha, \beta, \gamma}(p, q, s)\right)$.

3. $L_{x}^{\alpha} L_{y}^{\beta} L_{t}^{\gamma}\left(\left(\frac{t^{\gamma}}{\gamma}\right)^{n} u\left(\frac{x^{\alpha}}{\alpha}, \frac{y^{\beta}}{\beta}, \frac{t^{\gamma}}{\gamma}\right)\right)=(-1)^{n} \frac{\mathrm{d}^{n}}{\mathrm{~d} s^{n}}\left(U_{\alpha, \beta, \gamma}(p, q, s)\right)$.

4. $L_{x}^{\alpha} L_{y}^{\beta} L_{t}^{\gamma}\left(\left(\frac{x^{\alpha}}{\alpha}\right)^{l} \frac{\partial^{\beta+\gamma}}{\partial y^{\beta} \partial t \gamma}\left(u\left(\frac{x^{\alpha}}{\alpha}, \frac{y^{\beta}}{\beta}, \frac{t^{\gamma}}{\gamma}\right)\right)\right)=(-1)^{l} \frac{\mathrm{d}^{l}}{\mathrm{~d} p^{l}}\left(L_{x}^{\alpha} L_{y}^{\beta} L_{t}^{\gamma}\left(\frac{\partial^{\beta+\gamma}}{\partial y^{\beta} \partial t \gamma}\left(u\left(\frac{x^{\alpha}}{\alpha}, \frac{y^{\beta}}{\beta}, \frac{t^{\gamma}}{\gamma}\right)\right)\right)\right)$.

Proof. It can be easily verified with the help of definition of triple Laplace transform.

Theorem 3.4. For $\alpha, \beta, \gamma \in(0,1]$. Let $u\left(\frac{x^{\alpha}}{\alpha}, \frac{y^{\beta}}{\beta}, \frac{t^{\gamma}}{\gamma}\right)$ be the real valued piece-wise continuous function defined on the domain $(0, \infty) \times(0, \infty) \times(0, \infty)$. The FCTLT (Fractional Conformable triple Laplace transform) of the conformable partial fractional derivatives of order $\alpha, \beta$, and $\gamma$, then

1. $L_{x}^{\alpha} L_{y}^{\beta} L_{t}^{\gamma}\left(\frac{\partial^{\alpha}}{\partial x^{\alpha}}\left(u\left(\frac{x^{\alpha}}{\alpha}, \frac{y^{\beta}}{\beta}, \frac{t^{\gamma}}{\gamma}\right)\right)\right)=p U(p, q, s)-U(0, q, s)$.

2. $L_{x}^{\alpha} L_{y}^{\beta} L_{t}^{\gamma}\left(\frac{\partial^{\beta}}{\partial y^{\beta}}\left(u\left(\frac{x^{\alpha}}{\alpha}, \frac{y^{\beta}}{\beta}, \frac{t^{\gamma}}{\gamma}\right)\right)\right)=q U(p, q, s)-U(p, 0, s)$. 
3. $L_{x}^{\alpha} L_{y}^{\beta} L_{t}^{\gamma}\left(\frac{\partial^{\gamma}}{\partial t^{\gamma}}\left(u\left(\frac{x^{\alpha}}{\alpha}, \frac{y^{\beta}}{\beta}, \frac{t^{\gamma}}{\gamma}\right)\right)\right)=s U(p, q, s)-U(p, q, 0)$.

4. $L_{x}^{\alpha} L_{y}^{\beta} L_{t}^{\gamma}\left(\frac{\partial^{2 \alpha}}{\partial x^{2 \alpha}}\left(u\left(\frac{x^{\alpha}}{\alpha}, \frac{y^{\beta}}{\beta}, \frac{t^{\gamma}}{\gamma}\right)\right)\right)=p^{2} U(p, q, s)-p U(0, q, s)-U_{x}(0, q, s)$.

5. $L_{x}^{\alpha} L_{y}^{\beta} L_{t}^{\gamma}\left(\frac{\partial^{2 \beta}}{\partial y^{2 \beta}}\left(u\left(\frac{x^{\alpha}}{\alpha}, \frac{y^{\beta}}{\beta}, \frac{t^{\gamma}}{\gamma}\right)\right)\right)=q^{2} U(p, q, s)-q U(p, 0, s)-U_{y}(p, 0, s)$.

6. $L_{x}^{\alpha} L_{y}^{\beta} L_{t}^{\gamma}\left(\frac{\partial^{2 \gamma}}{\partial t^{2 \gamma}}\left(u\left(\frac{x^{\alpha}}{\alpha}, \frac{y^{\beta}}{\beta}, \frac{t^{\gamma}}{\gamma}\right)\right)\right)=s^{2} U(p, q, s)-s U(p, q, 0)-U_{t}(p, q, 0)$.

7. $L_{x}^{\alpha} L_{y}^{\beta} L_{t}^{\gamma}\left(\frac{\partial^{3 \alpha}}{\partial x^{3 \alpha}}\left(u\left(\frac{x^{\alpha}}{\alpha}, \frac{y^{\beta}}{\beta}, \frac{t^{\gamma}}{\gamma}\right)\right)\right)=p^{3} U(p, q, s)-p^{2} U(0, q, s)-p U_{x}(0, q, s)-U_{x x}(0, q, s)$.

Proof. Here we go for proof of result (1), and the remaining results (2-7) can be proved.

To obtain fractional conformable Triple Laplace transform of the fractional partial derivatives, we use integration by parts and Theorem 2.1.

By applying the definition of FCTLT, we have

$$
\begin{aligned}
& L_{x}^{\alpha} L_{y}^{\beta} L_{t}^{\gamma}\left(\frac{\partial^{\alpha}}{\partial x^{\alpha}}\left(u\left(\frac{x^{\alpha}}{\alpha}, \frac{y^{\beta}}{\beta}, \frac{t^{\gamma}}{\gamma}\right)\right)\right) \\
= & \int_{0}^{\infty} \int_{0}^{\infty} \int_{0}^{\infty} e^{-p\left(\frac{x^{\alpha}}{a}\right)-q\left(\frac{y^{\beta}}{\beta}\right)-s\left(\frac{t^{\gamma}}{\gamma}\right)} \frac{\partial^{\alpha}(u)}{\partial x^{\alpha}} x^{\alpha-1} y^{\beta-1} t^{\gamma-1} \mathrm{~d} x \mathrm{~d} y \mathrm{~d} t .
\end{aligned}
$$

Since we have Theorem 2.1, $\frac{\partial^{\alpha}(u)}{\partial x^{\alpha}}=x^{1-\alpha} \frac{\partial u}{\partial x}$. We use this result in to Eq. (3.4).

Therefore, Eq. (3.4) becomes,

$$
\begin{aligned}
& L_{x}^{\alpha} L_{y}^{\beta} L_{t}^{\gamma}\left(\frac{\partial^{\alpha}}{\partial x^{\alpha}}\left(u\left(\frac{x^{\alpha}}{\alpha}, \frac{y^{\beta}}{\beta}, \frac{t^{\gamma}}{\gamma}\right)\right)\right) \\
= & \int_{0}^{\infty} \int_{0}^{\infty} e^{-q\left(\frac{y^{\beta}}{\beta}\right)-s\left(\frac{t^{\gamma}}{\gamma}\right)}\left(\int_{0}^{\infty} e^{-p\left(\frac{x^{\alpha}}{\alpha}\right)} \frac{\partial u}{\partial x} \mathrm{~d} x\right) y^{\beta-1} t^{\gamma-1} \mathrm{~d} y \mathrm{~d} t .
\end{aligned}
$$

The integral inside the bracket is given by,

$$
\int_{0}^{\infty} e^{-p\left(\frac{x^{\alpha}}{\alpha}\right)} \frac{\partial u}{\partial x} \mathrm{~d} x=p U(p, y, t)-U(0, y, t) .
$$

By substituting Eq. (3.6) in Eq. (3.5), and simplifying, we get the required result (1). That is,

$$
L_{x}^{\alpha} L_{y}^{\beta} L_{t}^{\gamma}\left(\frac{\partial^{\alpha}}{\partial x^{\alpha}}\left(u\left(\frac{x^{\alpha}}{\alpha}, \frac{y^{\beta}}{\beta}, \frac{t^{\gamma}}{\gamma}\right)\right)\right)=p U(p, q, s)-U(0, q, s) .
$$

This completes the proof of the theorem. 
In general, the above results can be extended as,

$$
L_{x}^{\alpha} L_{y}^{\beta} L_{t}^{\gamma}\left(\frac{\partial^{\alpha+\beta+\gamma}}{\partial x^{\alpha} \partial y^{\beta} \partial t \gamma}\left(u\left(\frac{x^{\alpha}}{\alpha}, \frac{y^{\beta}}{\beta}, \frac{t^{\gamma}}{\gamma}\right)\right)\right)=\left(\begin{array}{c}
p q s U(p, q, s)-p U(p, 0,0) \\
-q U(0, q, 0)-s U(0,0, s) \\
-p q U(p, q, 0)-p s U(p, 0, s) \\
-q s U(0, q, s)-U(0,0,0)
\end{array}\right)
$$

and

$$
L_{x}^{\alpha} L_{y}^{\beta} L_{t}^{\gamma}\left(\frac{\partial^{m}}{\partial t^{m}}\left(u\left(\frac{x^{\alpha}}{\alpha}, \frac{y^{\beta}}{\beta}, \frac{t^{\gamma}}{\gamma}\right)\right)\right)=s^{m} U(p, q, s)-\sum_{k=0}^{m-1} s^{m-1-k} U_{t}^{(k)}(p, q, 0) .
$$

\section{Some results and theorems of the fractional conformable triple Sumudu transform}

In this section, we recall definition of Sumudu transform and defined conformable triple Sumudu transform and some important results on it and based on these results we will introduce new method to solve linear and nonlinear dynamic Partial fractional differential equation.

Definition 4.1. The conformable fractional Sumudu Transform (CFST) of function $u\left(\frac{x^{\alpha}}{\alpha}\right)$ is defined over the set,

$$
\mathcal{A}_{1}=\left\{u\left(\frac{x^{\alpha}}{\alpha}\right)\left|\exists K, \tau_{1}, \tau_{2}>0,\right| u(x) \mid<K e^{\frac{\left|\frac{x^{\alpha}}{\alpha}\right|}{\tau_{j}}}, \text { if } x^{\alpha} \epsilon(-1)^{j} \times[0, \infty)\right\} .
$$

And defined by the integral,

$$
\bar{u}_{\alpha}(p)=S_{x}^{\alpha}\left(u\left(\frac{x^{\alpha}}{\alpha}\right)\right)=\int_{0}^{\infty} e^{-\left(\frac{x^{\alpha}}{\alpha}\right)} u\left(p \frac{x^{\alpha}}{\alpha}\right) x^{\alpha-1} \mathrm{~d} x, \quad \frac{x^{\alpha}}{\alpha}>0, u \in\left(-\tau_{1}, \tau_{2}\right), \alpha \in(0,1] .
$$

Or equivalently,

$$
\bar{u}_{\alpha}(p)=S_{x}^{\alpha}\left(u\left(\frac{x^{\alpha}}{\alpha}\right)\right)=\frac{1}{p} \int_{0}^{\infty} e^{-\left(\frac{x^{\alpha}}{p \alpha}\right)} u\left(\frac{x^{\alpha}}{\alpha}\right) x^{\alpha-1} \mathrm{~d} x<\infty,
$$

where $p \in \mathbb{C}$ is the Sumudu variable correspond to $x$.

Definition 4.2. ([32]) Let $u\left(\frac{x^{\alpha}}{\alpha}, \frac{y^{\beta}}{\beta}\right)$ be a piecewise continuous function on the domain $D$ of $R^{+} \times R^{+}$. Then conformable fractional double Sumudu Transform (CFDST) of $u\left(\frac{x^{\alpha}}{\alpha}, \frac{y^{\beta}}{\beta}\right)$ is defined over the set: 


$$
\mathcal{A}_{2}=\left\{\begin{array}{c}
u\left(\frac{x^{\alpha}}{\alpha}, \frac{y^{\beta}}{\beta}\right)\left|\exists K, \tau_{1}, \tau_{2}>0,\right| u\left(\frac{x^{\alpha}}{\alpha}, \frac{y^{\beta}}{\beta}\right) \mid<K \exp \left(\frac{\left|\frac{x^{\alpha}}{\alpha}+\frac{y^{\beta}}{\beta}\right|}{\tau_{j}^{2}}\right) \\
\text { if } j=1,2 \text { and }\left(x^{\alpha}, y^{\beta}\right) \in R_{+}^{2}
\end{array}\right\} .
$$

By the integral,

$$
\begin{aligned}
\bar{u}_{\alpha, \beta}(p, q) & =S_{x}^{\alpha} S_{y}^{\beta}\left(u\left(\frac{x^{\alpha}}{\alpha}, \frac{y^{\beta}}{\beta}\right):(p, q)\right)=S_{x, y}^{\alpha, \beta}\left(u\left(\frac{x^{\alpha}}{\alpha}, \frac{y^{\beta}}{\beta}\right):(p, q)\right) \\
& =\int_{0}^{\infty} \int_{0}^{\infty} e^{-\left(\frac{x^{\alpha}}{\alpha}+\frac{y^{\beta}}{\beta}\right)} u\left(p \frac{x^{\alpha}}{\alpha}, q \frac{y^{\beta}}{\beta}\right) x^{\alpha-1} y^{\beta-1} \mathrm{~d} x \mathrm{~d} y,
\end{aligned}
$$

where $\frac{x^{\alpha}}{\alpha}, \frac{y^{\beta}}{\beta}>0$, and $p, q$ are the transform variable of $x$ and $y$ accordingly.

Definition 4.3. The fractional conformable partial Sumudu transformation of a piecewise continuous real valued function $u: R^{+} \times R^{+} \times R^{+} \rightarrow R$, is defined as follows and it can be extended over the set as it is mentioned in Eq. (4.1):

$$
\begin{aligned}
& \bar{u}_{\alpha}(p)=S_{x}^{\alpha}\left(u\left(\frac{x^{\alpha}}{\alpha}, \frac{y^{\beta}}{\beta}, \frac{t^{\gamma}}{\gamma}\right): p\right)=\int_{0}^{\infty} e^{-\frac{x^{\alpha}}{\alpha}} u\left(p \frac{x^{\alpha}}{\alpha}, \frac{y^{\beta}}{\beta}, \frac{t^{\gamma}}{\gamma}\right) x^{\alpha-1} \mathrm{~d} x, \\
& \bar{u}_{\beta}(q)=S_{y}^{\beta}\left(u\left(\frac{x^{\alpha}}{\alpha}, \frac{y^{\beta}}{\beta}, \frac{t^{\gamma}}{\gamma}\right): q\right)=\int_{0}^{\infty} e^{-\frac{y^{\beta}}{\beta}} u\left(\frac{x^{\alpha}}{\alpha}, q \frac{y^{\beta}}{\beta}, \frac{t^{\gamma}}{\gamma}\right) y^{\beta-1} \mathrm{~d} y, \\
& \bar{u}_{\gamma}(s)=S_{t}^{\gamma}\left(u\left(\frac{x^{\alpha}}{\alpha}, \frac{y^{\beta}}{\beta}, \frac{t^{\gamma}}{\gamma}\right): s\right)=\int_{0}^{\infty} e^{-\frac{x^{\gamma}}{\gamma}} u\left(\frac{x^{\alpha}}{\alpha}, \frac{y^{\beta}}{\beta}, s \frac{t^{\gamma}}{\gamma}\right) t^{\gamma-1} \mathrm{~d} t .
\end{aligned}
$$

Now, we define Conformable triple Sumudu transform, for $\alpha, \beta, \gamma \in(0,1]$, and $p, q, s \in \mathbb{C}$ are the transformable or Sumudu variables of positive $x, y$ and $t$ respectively.

Definition 4.4. Let $u\left(\frac{x^{\alpha}}{\alpha}, \frac{y^{\beta}}{\beta}, \frac{t^{\gamma}}{\gamma}\right)$ be a real valued piece wise continuous function defined on the domain $D$ of $R^{+} \times R^{+} \times R^{+}$of exponential order. Then, the fractional conformable triple Sumudu transform (FCTST) of $u\left(\frac{x^{\alpha}}{\alpha}, \frac{y^{\beta}}{\beta}, \frac{t^{\gamma}}{\gamma}\right)$ is defined as follows:

$$
\begin{aligned}
& \bar{u}_{\alpha, \beta, \gamma}(p, q, s)=S_{x, y, t}^{\alpha, \beta, \gamma}\left(u\left(\frac{x^{\alpha}}{\alpha}, \frac{y^{\beta}}{\beta}, \frac{t^{\gamma}}{\gamma}\right):(p, q, s)\right) \\
= & \int_{0}^{\infty} \int_{0}^{\infty} \int_{0}^{\infty} e^{-\left(\frac{x^{\alpha}}{\alpha}\right)-\left(\frac{y^{\beta}}{\beta}\right)-\left(\frac{t^{\gamma}}{\gamma}\right)} u\left(p \frac{x^{\alpha}}{\alpha}, q \frac{y^{\beta}}{\beta}, s \frac{t^{\gamma}}{\gamma}\right) x^{\alpha-1} y^{\beta-1} t^{\gamma-1} \mathrm{~d} x \mathrm{~d} y \mathrm{~d} t .
\end{aligned}
$$

The conformable inverse triple Sumudu transform is defined by,

$$
u\left(\frac{x^{\alpha}}{\alpha}, \frac{y^{\beta} p}{\beta}, \frac{t^{\gamma}}{\gamma}\right)=S^{-1}\left(\bar{u}_{\alpha, \beta, \gamma}(p, q, s)\right)
$$




$$
=\frac{1}{2 \pi i} \int_{\alpha-i \infty}^{\alpha+i \infty} e^{\left(\frac{x^{\alpha}}{\alpha}\right)}\left[\frac{1}{2 \pi i} \int_{\beta-i \infty}^{\beta+\infty} e^{\left(\frac{y^{\beta}}{\beta}\right)}\left[\frac{1}{2 \pi i} \int_{\gamma-i \infty}^{\gamma+i \infty} e^{\left(\frac{t^{\gamma}}{\gamma}\right)} \bar{u}_{\alpha, \beta, \gamma}(p, q, s) \mathrm{d} s\right] \mathrm{d} q\right] \mathrm{d} p .
$$

Remark 4.1. The existence condition for the Conformable triple Sumudu transform.

If $u\left(\frac{x^{\alpha}}{\alpha}, \frac{y^{\beta}}{\beta}, \frac{t^{\gamma}}{\gamma}\right)$ is an exponential order $a, b$ and $c$ then there exist $K>0$, for all $\frac{x^{\alpha}}{\alpha}>X$, $\frac{y^{\beta}}{\beta}>Y, \frac{t \gamma}{\gamma}>T$, such that

$$
\left|u\left(\frac{x^{\alpha}}{\alpha}, \frac{y^{\beta}}{\beta}, \frac{t^{\gamma}}{\gamma}\right)\right| \leq \operatorname{Kexp}\left(a\left(\frac{x^{\alpha}}{\alpha}\right)+b\left(\frac{y^{\beta}}{\beta}\right)+c\left(\frac{t^{\gamma}}{\gamma}\right)\right) .
$$

And we write,

$$
\begin{gathered}
u\left(\frac{x^{\alpha}}{\alpha}, \frac{y^{\beta}}{\beta}, \frac{t^{\gamma}}{\gamma}\right)=O\left(\exp \left(a\left(\frac{x^{\alpha}}{\alpha}\right)+b\left(\frac{y^{\beta}}{\beta}\right)+c\left(\frac{t^{\gamma}}{\gamma}\right)\right)\right) \\
\text { as }\left(\frac{x^{\alpha}}{\alpha}, \frac{y^{\beta}}{\beta}, \frac{t^{\gamma}}{\gamma}\right) \rightarrow(\infty, \infty, \infty) .
\end{gathered}
$$

Equivalently,

$$
\begin{aligned}
& \lim \left(\frac{x^{\alpha}}{\alpha}, \frac{y^{\beta}}{\beta}, \frac{r}{\gamma}\right) \rightarrow(\infty, \infty, \infty) \\
= & K \text { (Finite quantity). }
\end{aligned}
$$

It means, the function $u\left(\frac{x^{\alpha}}{\alpha}, \frac{y^{\beta}}{\beta}, \frac{t \gamma}{\gamma}\right)$ does not grow faster than $K \mathrm{e}^{\left(a\left(\frac{x^{\alpha}}{\alpha}\right)+b\left(\frac{y^{\beta}}{\beta}\right)+c\left(\frac{t^{\gamma}}{\gamma}\right)\right)}$ as $\left(\frac{x^{\alpha}}{\alpha}, \frac{y^{\beta}}{\beta}, \frac{t^{\gamma}}{\gamma}\right) \rightarrow(\infty, \infty, \infty)$.

Theorem 4.1. Let $u$ and $v: R^{+} \rightarrow R$ be the given functions, and for $\alpha, \beta, \gamma \in(0,1]$.

$$
\text { If } \left.S_{x}^{\alpha} S_{y}^{\beta} S_{t}^{\gamma}\left(u\left(\frac{x^{\alpha}}{\alpha}, \frac{y^{\beta}}{\beta}, \frac{t^{\gamma}}{\gamma}\right): p, q, s\right)\right)=\bar{u}_{\alpha, \beta, \gamma}(p, q, s), S_{x}^{\alpha} S_{y}^{\beta} S_{t}^{\gamma}\left(v\left(\frac{x^{\alpha}}{\alpha}, \frac{y^{\beta}}{\beta}, \frac{t^{\gamma}}{\gamma}\right)\right)=\bar{v}_{\alpha, \beta, \gamma}(p, q, s)
$$
and $A, B$ and $C$ are constants. Then the conformable triple Sumudu transform of some functions are given by,

1. Linearity property:

$$
\begin{aligned}
& S_{x}^{\alpha} S_{y}^{\beta} S_{t}^{\gamma}\left(A u\left(\frac{x^{\alpha}}{\alpha}, \frac{y^{\beta}}{\beta}, \frac{t^{\gamma}}{\gamma}\right)+B v\left(\frac{x^{\alpha}}{\alpha}, \frac{y^{\beta}}{\beta}, \frac{t^{\gamma}}{\gamma}\right):(p, q, s)\right) \\
& =A S_{x}^{\alpha} S_{y}^{\beta} S_{t}^{\gamma}\left(u\left(\frac{x^{\alpha}}{\alpha}, \frac{y^{\beta}}{\beta}, \frac{t^{\gamma}}{\gamma}\right):(p, q, s)\right)+B S_{x}^{\alpha} S_{y}^{\beta} S_{t}^{\gamma}\left(v\left(\frac{x^{\alpha}}{\alpha}, \frac{y^{\beta}}{\beta}, \frac{t^{\gamma}}{\gamma}\right):(p, q, s)\right) \\
& =A \bar{u}_{\alpha, \beta, \gamma}(p, q, s)+B \bar{v}_{\alpha, \beta, \gamma}(p, q, s) .
\end{aligned}
$$

2. $S_{x}^{\alpha} S_{y}^{\beta} S_{t}^{\gamma}(C:(p, q, s))=C$, where $C$ is the constant. 
3. $S_{x}^{\alpha} S_{y}^{\beta} S_{t}^{\gamma}\left(\left(\frac{x^{\alpha}}{\alpha}\right)^{l}\left(\frac{y^{\beta}}{\beta}\right)^{m}\left(\frac{t^{\gamma}}{\gamma}\right)^{n}:(p, q, s)\right)=\Gamma(l+1) \Gamma(m+1) \Gamma(n+1) p^{l} q^{m} s^{n}$, where $\Gamma(\cdot)$ is the gamma function. Note that $\Gamma(n+1)=n !$, for $\mathrm{n}=0,1,2,3, \ldots$

4. $S_{x}^{\alpha} S_{y}^{\beta} S_{t}^{\gamma}\left(\exp \left(A \frac{x^{\alpha}}{\alpha}+B \frac{y^{\beta}}{\beta}+C \frac{t^{\gamma}}{\gamma}\right):(p, q, s)\right)=\frac{1}{(1-A p)(1-B q)(1-C s)}$.

5. $S_{x}^{\alpha} S_{y}^{\beta} S_{t}^{\gamma}\left(u\left(\frac{x^{\alpha}}{\alpha}, \frac{y^{\beta}}{\beta}, \frac{t^{\gamma}}{\gamma}\right) v\left(\frac{x^{\alpha}}{\alpha}, \frac{y^{\beta}}{\beta}, \frac{t^{\gamma}}{\gamma}\right):(p, q, s)\right)=\bar{u}_{\alpha, \beta, \gamma}(p, q, s) \bar{v}_{\alpha, \beta, \gamma}(p, q, s)$.

6. $S_{x}^{\alpha} S_{y}^{\beta} S_{t}^{\gamma}\left(\sin \left(A \frac{x^{\alpha}}{\alpha}\right) \sin \left(B \frac{y^{\beta}}{\beta}\right) \sin \left(C \frac{t^{\gamma}}{\gamma}\right):(p, q, s)\right)=\frac{A B C p q s}{\left(1+p^{2} A^{2}\right)\left(1+q^{2} B^{2}\right)\left(1+s^{2} C^{2}\right)^{\prime}}$,

$S_{x}^{\alpha} S_{y}^{\beta} S_{t}^{\gamma}\left(\cos \left(A \frac{x^{\alpha}}{\alpha}\right) \cos \left(B \frac{y^{\beta}}{\beta}\right) \cos \left(C \frac{t^{\gamma}}{\gamma}\right)\right)=\frac{1}{\left(1+p^{2} A^{2}\right)\left(1+q^{2} B^{2}\right)\left(1+s^{2} C^{2}\right)}$.

Proof. It can be verify with the help of definition of Triple Sumudu Transform.

Theorem 4.2. If $S_{x}^{\alpha} S_{y}^{\beta} S_{t}^{\gamma}\left(u\left(\frac{x^{\alpha}}{\alpha}, \frac{y^{\beta}}{\beta}, \frac{t^{\gamma}}{\gamma}\right)\right)=\bar{u}_{\alpha, \beta, \gamma}(p, q, s)=\bar{u}(p, q, s)$, then the conformable fractional triple Sumudu Transform of the functions, $\frac{x^{\alpha}}{\alpha} u(x, y, t), \frac{y^{\beta}}{\beta} u\left(\frac{x^{\alpha}}{\alpha}, \frac{y^{\beta}}{\beta}, \frac{t^{\gamma}}{\gamma}\right), \frac{t^{\gamma}}{\gamma} u\left(\frac{x^{\alpha}}{\alpha}, \frac{y^{\beta}}{\beta}, \frac{t^{\gamma}}{\gamma}\right)$, $\left(\frac{x^{\alpha}}{\alpha}\right)^{2} u(x, y, t), \frac{x^{\alpha}}{\alpha} \frac{y^{\beta}}{\beta} u(x, y, t), \frac{x^{\alpha}}{\alpha} \frac{y^{\beta}}{\beta} \frac{t^{\gamma}}{\gamma} u(x, y, t)$ are given by,

1. $S_{x}^{\alpha} S_{y}^{\beta} S_{t}^{\gamma}\left(\frac{x^{\alpha}}{\alpha} u\left(\frac{x^{\alpha}}{\alpha}, \frac{y^{\beta}}{\beta}, \frac{t^{\gamma}}{\gamma}\right)\right)=p^{2} \frac{\partial \bar{u}(p, q, s)}{\partial p}+p \bar{u}(p, q, s)$.

2. $S_{x}^{\alpha} S_{y}^{\beta} S_{t}^{\gamma}\left(\frac{y^{\beta}}{\beta} u\left(\frac{x^{\alpha}}{\alpha}, \frac{y^{\beta}}{\beta}, \frac{t^{\gamma}}{\gamma}\right)\right)=q^{2} \frac{\partial \bar{u}(p, q, s)}{\partial q}+q \bar{u}(p, q, s)$.

3. $S_{x}^{\alpha} S_{y}^{\beta} S_{t}^{\gamma}\left(\frac{t^{\gamma}}{\gamma} u\left(\frac{x^{\alpha}}{\alpha}, \frac{y^{\beta}}{\beta}, \frac{t^{\gamma}}{\gamma}\right)\right)=s^{2} \frac{\partial \bar{u}(p, q, s)}{\partial s}+s \bar{u}(p, q, s)$.

4. $S_{x}^{\alpha} S_{y}^{\beta} S_{t}^{\gamma}\left(\left(\frac{x^{\alpha}}{\alpha}\right)^{2} u\left(\frac{x^{\alpha}}{\alpha}, \frac{y^{\beta}}{\beta}, \frac{t^{\gamma}}{\gamma}\right)\right)=p^{4} \frac{\partial^{2} \bar{u}(p, q, s)}{\partial p^{2}}+4 p^{3} \frac{\partial \bar{u}(p, q, s)}{\partial p}+2 p^{2} \bar{u}(p, q, s)$.

5. $S_{x}^{\alpha} S_{y}^{\beta} S_{t}^{\gamma}\left(\frac{x^{\alpha}}{\alpha} \frac{y^{\beta}}{\beta} u\left(\frac{x^{\alpha}}{\alpha}, \frac{y^{\beta}}{\beta}, \frac{t^{\gamma}}{\gamma}\right)\right)=p^{2} q^{2} \frac{\partial^{2} \bar{u}(p, q, s)}{\partial p \partial q}+p^{2} q \frac{\partial \bar{u}(p, q, s)}{\partial p}+p q^{2} \frac{\partial \bar{u}(p, q, s)}{\partial q}$ $+p q \bar{u}(p, q, s)$.

6. $S_{x}^{\alpha} S_{y}^{\beta} S_{t}^{\gamma}\left(\frac{x^{\alpha}}{\alpha} \frac{y^{\beta}}{\beta} \frac{t^{\gamma}}{\gamma} u\left(\frac{x^{\alpha}}{\alpha}, \frac{y^{\beta}}{\beta}, \frac{t^{\gamma}}{\gamma}\right)\right)=p^{2} q^{2} s^{2} \frac{\partial^{3} \bar{u}(p, q, s)}{\partial p \partial q \partial s}+p q^{2} s^{2} \frac{\partial^{2} \bar{u}(p, q, s)}{\partial q \partial s}$ $+p^{2} q s^{2} \frac{\partial^{2} \bar{u}(p, q, s)}{\partial p \partial s}+p^{2} q^{2} s \frac{\partial^{2} \bar{u}(p, q, s)}{\partial p q}+p^{2} q s \frac{\partial \bar{u}(p, q, s)}{\partial p}$ $+p q^{2} s \frac{\partial \bar{u}(p, q, s)}{\partial q}+p q s^{2} \frac{\partial \bar{u}(p, q, s)}{\partial s}+p q s \bar{u}(p, q, s)$. 
Proof. Here we prove results 1, 4, and 6. Remaining can be proved similar manner.

By definition of Fractional Conformable triple Sumudu transform, we have,

$$
\begin{aligned}
& \bar{u}(p, q, s)=S_{x, y, t}^{\alpha, \beta, \gamma}\left(u\left(\frac{x^{\alpha}}{\alpha}, \frac{y^{\beta}}{\beta}, \frac{t^{\gamma}}{r}\right)\right) \\
= & \frac{1}{p q s} \int_{0}^{\infty} \int_{0}^{\infty} \int_{0}^{\infty} e^{-\left(\frac{x^{\alpha}}{p \alpha}\right)-\left(\frac{y^{\beta}}{q \beta}\right)-\left(\frac{t^{\gamma}}{s \gamma}\right)} u\left(\frac{x^{\alpha}}{\alpha}, \frac{y^{\beta}}{\beta}, \frac{t^{\gamma}}{\gamma}\right) x^{\alpha-1} y^{\beta-1} t^{\gamma-1} \mathrm{~d} x \mathrm{~d} y \mathrm{~d} t .
\end{aligned}
$$

Differentiating Eq. (4.7) with respect to ' $p$ ',

$$
\begin{aligned}
\frac{\partial \bar{u}}{\partial p}=\frac{1}{q S} \int_{0}^{\infty} \int_{0}^{\infty} e^{-\left(\frac{y^{\beta}}{q \beta}\right)-\left(\frac{t^{\gamma}}{s \gamma}\right)}\left(\int_{0}^{\infty} \frac{\partial}{\partial p}\left(\frac{1}{p} e^{-\left(\frac{x^{\alpha}}{p \alpha}\right)}\right) u\left(\frac{x^{\alpha}}{\alpha}, \frac{y^{\beta}}{\beta}, \frac{t^{\gamma}}{\gamma}\right) x^{\alpha-1} \mathrm{~d} x\right) \\
\times y^{\beta-1} t^{\gamma-1} \mathrm{~d} y \mathrm{~d} t .
\end{aligned}
$$

We partially differentiate inside the brackets,

$$
\begin{aligned}
& \int_{0}^{\infty} \frac{\partial}{\partial p}\left(\frac{1}{p} e^{-\left(\frac{x^{\alpha}}{p \alpha}\right)}\right) u\left(\frac{x^{\alpha}}{\alpha}, \frac{y^{\beta}}{\beta}, \frac{t^{\gamma}}{\gamma}\right) x^{\alpha-1} \mathrm{~d} x=\int_{0}^{\infty}\left(\begin{array}{c}
\left(\frac{1}{p^{3}} \frac{x^{\alpha}}{\alpha}-\frac{1}{p^{2}}\right) e^{-\left(\frac{x^{\alpha}}{p \alpha}\right)} \\
u\left(\frac{x^{\alpha}}{\alpha}, \frac{y^{\beta}}{\beta}, \frac{t^{\gamma}}{\gamma}\right)
\end{array}\right) x^{\alpha-1} \mathrm{~d} x \\
= & \int_{0}^{\infty} \frac{1}{p^{3}} e^{-\left(\frac{x^{\alpha}}{p \alpha}\right)} \frac{x^{\alpha}}{\alpha} u\left(\frac{x^{\alpha}}{\alpha}, \frac{y^{\beta}}{\beta}, \frac{t^{\gamma}}{\gamma}\right) x^{\alpha-1} \mathrm{~d} x-\int_{0}^{\infty} \frac{1}{p^{2}} e^{-\left(\frac{x^{\alpha}}{p \alpha}\right)} u\left(\frac{x^{\alpha}}{\alpha}, \frac{y^{\beta}}{\beta}, \frac{t^{\gamma}}{\gamma}\right) x^{\alpha-1} \mathrm{~d} x .
\end{aligned}
$$

Substituting Eq. (4.9) in Eq. (4.8). we have,

$$
\begin{gathered}
\frac{\partial \bar{u}(p, q, s)}{\partial p}=\frac{1}{p^{3} q s} \int_{0}^{\infty} \int_{0}^{\infty} \int_{0}^{\infty} e^{-\left(\frac{x^{\alpha}}{p \alpha}\right)-\left(\frac{y^{\beta}}{q \beta}\right)-\left(\frac{t^{\gamma}}{s \gamma}\right)} \frac{x^{\alpha}}{\alpha} u\left(\frac{x^{\alpha}}{\alpha}, \frac{y^{\beta}}{\beta}, \frac{t^{\gamma}}{\gamma}\right) x^{\alpha-1} y^{\beta-1} t^{\gamma-1} \mathrm{~d} x \mathrm{~d} y \mathrm{~d} t \\
\quad-\frac{1}{p^{2} q s} \int_{0}^{\infty} \int_{0}^{\infty} \int_{0}^{\infty} e^{-\left(\frac{x^{\alpha}}{p \alpha}\right)-\left(\frac{y^{\beta}}{q \beta}\right)-\left(\frac{t^{\gamma}}{s \gamma}\right)} u\left(\frac{x^{\alpha}}{\alpha}, \frac{y^{\beta}}{\beta}, \frac{t^{\gamma}}{\gamma}\right) x^{\alpha-1} y^{\beta-1} t^{\gamma-1} \mathrm{~d} x \mathrm{~d} y \mathrm{~d} t, \\
\frac{\partial \bar{u}(p, q, s)}{\partial p}=\frac{1}{p^{2}} S_{x}^{\alpha} S_{y}^{\beta} S_{t}^{\gamma}\left(\frac{x^{\alpha}}{\alpha} u\left(\frac{x^{\alpha}}{\alpha}, \frac{y^{\beta}}{\beta}, \frac{t^{\gamma}}{\gamma}\right)\right)-\frac{1}{p} S_{x}^{\alpha} S_{y}^{\beta} S_{t}^{\gamma}\left(u\left(\frac{x^{\alpha}}{\alpha}, \frac{y^{\beta}}{\beta}, \frac{t^{\gamma}}{\gamma}\right)\right),
\end{gathered}
$$

which gives,

$$
S_{x}^{\alpha} S_{y}^{\beta} S_{t}^{\gamma}\left(\frac{x^{\alpha}}{\alpha} u\left(\frac{x^{\alpha}}{\alpha}, \frac{y^{\beta}}{\beta}, \frac{t^{\gamma}}{\gamma}\right)\right)=p^{2} \frac{\partial \bar{u}(p, q, s)}{\partial p}+p \bar{u} .
$$

Hence the result (1) proved. To prove result (4), we need to partially differentiate of Eq. (4.7) with respect to ' $p$ ' twice. We can get,

$$
\begin{aligned}
& \frac{\partial^{2} \bar{u}(p, q, s)}{\partial p^{2}}=\frac{2}{p^{2}} \bar{u}-\frac{4}{p^{3}} S_{x}^{\alpha} S_{y}^{\beta} S_{t}^{\gamma}\left(\frac{x^{\alpha}}{\alpha} u\left(\frac{x^{\alpha}}{\alpha}, \frac{y^{\beta}}{\beta}, \frac{t^{\gamma}}{\gamma}\right)\right)+\frac{1}{p^{4}} S_{x}^{\alpha} S_{y}^{\beta} S_{t}^{\gamma}\left(\left(\frac{x^{\alpha}}{\alpha}\right)^{2} u\left(\frac{x^{\alpha}}{\alpha}, \frac{y^{\beta}}{\beta}, \frac{t^{\gamma}}{\gamma}\right)\right), \\
& S_{x}^{\alpha} S_{y}^{\beta} S_{t}^{\gamma}\left(\left(\frac{x^{\alpha}}{\alpha}\right)^{2} u\left(\frac{x^{\alpha}}{\alpha}, \frac{y^{\beta}}{\beta}, \frac{t^{\gamma}}{\gamma}\right)\right)=p^{4} \frac{\partial^{2} \bar{u}}{\partial p^{2}}-2 p^{2} \bar{u}+4 p S_{x}^{\alpha} S_{y}^{\beta} S_{t}^{\gamma}\left(\frac{x^{\alpha}}{\alpha} u\left(\frac{x^{\alpha}}{\alpha}, \frac{y^{\beta}}{\beta}, \frac{t^{\gamma}}{\gamma}\right)\right) .
\end{aligned}
$$


Using Eq. (4.7) in Eq. (4.11) and simplifying, we have,

$$
S_{x}^{\alpha} S_{y}^{\beta} S_{t}^{\gamma}\left(\left(\frac{x^{\alpha}}{\alpha}\right)^{2} u\left(\frac{x^{\alpha}}{\alpha}, \frac{y^{\beta}}{\beta}, \frac{t^{\gamma}}{\gamma}\right)\right)=p^{4} \frac{\partial^{2} \bar{u}(p, q, s)}{\partial p^{2}}+4 p^{3} \frac{\partial \bar{u}(p, q, s)}{\partial p}+2 p^{2} \bar{u} .
$$

Hence the result (4) is proved.

Now to prove the result (6), we differentiate partially of Eq. (4.7) with respect to $p, q$ and $s$ respectively, we have,

$$
\begin{aligned}
& \frac{\partial^{3} \bar{u}(p, q, s)}{\partial p \partial q \partial s} \\
= & \frac{1}{p^{3} q^{3} s^{3}} \int_{0}^{\infty} \int_{0}^{\infty} \int_{0}^{\infty} e^{-\left(\frac{x^{\alpha}}{p \alpha}\right)-\left(\frac{y^{\beta}}{q \beta}\right)-\left(\frac{t \gamma}{s \gamma}\right)} \frac{x^{\alpha} y^{\beta}}{\alpha} \frac{t^{\gamma}}{\gamma} u\left(\frac{x^{\alpha}}{\alpha}, \frac{y^{\beta}}{\beta}, \frac{t^{\gamma}}{\gamma}\right) x^{\alpha-1} y^{\beta-1} t^{\gamma-1} \mathrm{~d} x \mathrm{~d} y \mathrm{~d} t \\
- & \frac{1}{p^{3} q^{3} s^{2}} \int_{0}^{\infty} \int_{0}^{\infty} \int_{0}^{\infty} e^{-\left(\frac{x^{\alpha}}{p \alpha}\right)-\left(\frac{y^{\beta}}{q \beta}\right)-\left(\frac{t^{\gamma}}{s \gamma}\right)} \frac{x^{\alpha}}{\alpha} \frac{y^{\beta}}{\beta} u\left(\frac{x^{\alpha}}{\alpha}, \frac{y^{\beta}}{\beta}, \frac{t^{\gamma}}{\gamma}\right) x^{\alpha-1} y^{\beta-1} t^{\gamma-1} \mathrm{~d} x \mathrm{~d} y \mathrm{~d} t \\
- & \frac{1}{p^{3} q^{2} s^{3}} \int_{0}^{\infty} \int_{0}^{\infty} \int_{0}^{\infty} e^{-\left(\frac{x^{\alpha}}{p \alpha}\right)-\left(\frac{y^{\beta}}{q \beta}\right)-\left(\frac{t^{\gamma}}{s \gamma}\right)} \frac{x^{\alpha}}{\alpha} \frac{t^{\gamma}}{\gamma} u\left(\frac{x^{\alpha}}{\alpha}, \frac{y^{\beta}}{\beta}, \frac{t^{\gamma}}{\gamma}\right) x^{\alpha-1} y^{\beta-1} t^{\gamma-1} \mathrm{~d} x \mathrm{~d} y \mathrm{~d} t \\
- & \frac{1}{p^{2} q^{3} s^{3}} \int_{0}^{\infty} \int_{0}^{\infty} \int_{0}^{\infty} e^{-\left(\frac{x^{\alpha}}{p \alpha}\right)-\left(\frac{y^{\beta}}{q \beta}\right)-\left(\frac{t^{\gamma}}{s \gamma}\right)} \frac{y^{\beta} t^{\gamma}}{\beta} u\left(\frac{x^{\alpha}}{\alpha}, \frac{y^{\beta}}{\beta}, \frac{t^{\gamma}}{\gamma}\right) x^{\alpha-1} y^{\beta-1} t^{\gamma-1} \mathrm{~d} x \mathrm{~d} y \mathrm{~d} t \\
+ & \frac{1}{p^{3} q^{2} s^{2}} \int_{0}^{\infty} \int_{0}^{\infty} \int_{0}^{\infty} e^{-\left(\frac{x^{\alpha}}{p \alpha}\right)-\left(\frac{y^{\beta}}{q \beta}\right)-\left(\frac{t^{\gamma}}{s \gamma}\right)} \frac{x^{\alpha}}{a} u\left(\frac{x^{\alpha}}{\alpha}, \frac{y^{\beta}}{\beta}, \frac{t^{\gamma}}{\gamma}\right) x^{\alpha-1} y^{\beta-1} t^{\gamma-1} \mathrm{~d} x \mathrm{~d} y \mathrm{~d} t \\
+ & \frac{1}{p^{2} q^{3} s^{2}} \int_{0}^{\infty} \int_{0}^{\infty} \int_{0}^{\infty} e^{-\left(\frac{x^{\alpha}}{p \alpha}\right)-\left(\frac{y^{\beta}}{q \beta}\right)-\left(\frac{t \gamma}{s \gamma}\right)} \frac{y^{\beta}}{\beta} u\left(\frac{x^{\alpha}}{\alpha}, \frac{y^{\beta}}{\beta}, \frac{t^{\gamma}}{\gamma}\right) x^{\alpha-1} y^{\beta-1} t^{\gamma-1} \mathrm{~d} x \mathrm{~d} y \mathrm{~d} t \\
+ & \frac{1}{p^{2} q^{2} s^{3}} \int_{0}^{\infty} \int_{0}^{\infty} \int_{0}^{\infty} e^{-\left(\frac{x^{\alpha}}{p \alpha}\right)-\left(\frac{y^{\beta}}{q \beta}\right)-\left(\frac{t^{\gamma}}{s \gamma}\right)} \frac{t^{\gamma}}{\gamma} u\left(\frac{x^{\alpha}}{a}, \frac{y^{\beta}}{\beta}, \frac{t^{\gamma}}{\gamma}\right) x^{\alpha-1} y^{\beta-1} t^{\gamma-1} \mathrm{~d} x \mathrm{~d} y \mathrm{~d} t \\
- & \frac{1}{p^{2} q^{2} s^{2}} \int_{0}^{\infty} \int_{0}^{\infty} \int_{0}^{\infty} e^{-\left(\frac{x^{\alpha}}{p \alpha}\right)-\left(\frac{y^{\beta}}{q \beta}\right)-\left(\frac{t^{\gamma}}{s \gamma}\right)} u\left(\frac{x^{\alpha}}{\alpha}, \frac{y^{\beta}}{\beta}, \frac{t^{\gamma}}{\gamma}\right) x^{\alpha-1} y^{\beta-1} t^{\gamma-1} \mathrm{~d} x \mathrm{~d} y \mathrm{~d} t .
\end{aligned}
$$

Therefore,

$$
\begin{aligned}
& \frac{\partial^{3} \bar{u}(p, q, s)}{\partial p \partial q \partial s}=\frac{1}{p^{2} q^{2} s^{2}} S_{x}^{\alpha} S_{y}^{\beta} S_{t}^{\gamma}\left(\frac{x^{\alpha}}{\alpha} \frac{y^{\beta}}{\beta} \frac{t^{\gamma}}{\gamma} u\left(\frac{x^{\alpha}}{\alpha}, \frac{y^{\beta}}{\beta}, \frac{t^{\gamma}}{\gamma}\right)\right) \\
& -\frac{1}{p^{2} q^{2} s} S_{x}^{\alpha} S_{y}^{\beta} S_{t}^{\gamma}\left(\frac{x^{\alpha}}{\alpha} \frac{y^{\beta}}{\beta} u\left(\frac{x^{\alpha}}{\alpha}, \frac{y^{\beta}}{\beta}, \frac{t^{\gamma}}{\gamma}\right)\right)-\frac{1}{p^{2} q s^{2}} S_{x}^{\alpha} S_{y}^{\beta} S_{t}^{\gamma}\left(\frac{x^{\alpha}}{\alpha} \frac{t^{\gamma}}{\gamma} u\left(\frac{x^{\alpha}}{\alpha}, \frac{y^{\beta}}{\beta}, \frac{t^{\gamma}}{\gamma}\right)\right) \\
& -\frac{1}{p q^{2} s^{2}} S_{x}^{\alpha} S_{y}^{\beta} S_{t}^{\gamma}\left(\frac{y^{\beta}}{\beta} \frac{t^{\gamma}}{\gamma} u\left(\frac{x^{\alpha}}{\alpha}, \frac{y^{\beta}}{\beta}, \frac{t^{\gamma}}{\gamma}\right)\right)+\frac{1}{p^{2} q s} S_{x}^{\alpha} S_{y}^{\beta} S_{t}^{\gamma}\left(\frac{x^{\alpha}}{\alpha} u\left(\frac{x^{\alpha}}{\alpha}, \frac{y^{\beta}}{\beta}, \frac{t^{\gamma}}{\gamma}\right)\right) \\
& +\frac{1}{p q^{2} s} S_{x}^{\alpha} S_{y}^{\beta} S_{t}^{\gamma}\left(\frac{y^{\beta}}{\beta} u\left(\frac{x^{\alpha}}{\alpha}, \frac{y^{\beta}}{\beta}, \frac{t^{\gamma}}{\gamma}\right)\right)+\frac{1}{p q s^{2}} S_{x}^{\alpha} S_{y}^{\beta} S_{t}^{\gamma}\left(\frac{t^{\gamma}}{\gamma} u\left(\frac{x^{\alpha}}{\alpha}, \frac{y^{\beta}}{\beta}, \frac{t^{\gamma}}{\gamma}\right)\right)
\end{aligned}
$$




$$
-\frac{1}{p q s} S_{x}^{\alpha} S_{y}^{\beta} S_{t}^{\gamma}\left(u\left(\frac{x^{\alpha}}{\alpha}, \frac{y^{\beta}}{\beta}, \frac{t^{\gamma}}{\gamma}\right)\right) .
$$

Using the results of Theorem 4.2 (Results -1-5) and Simplifying, we get,

$$
\begin{aligned}
& S_{x}^{\alpha} S_{y}^{\beta} S_{t}^{\gamma}\left(\frac{x^{\alpha}}{\alpha} \frac{y^{\beta}}{\beta} \frac{t^{\gamma}}{\gamma} u\left(\frac{x^{\alpha}}{\alpha}, \frac{y^{\beta}}{\beta}, \frac{t^{\gamma}}{\gamma}\right)\right) \\
= & p^{2} q^{2} s^{2} \frac{\partial^{3} \bar{u}(p, q, s)}{\partial p \partial q \partial s}+p q^{2} s^{2} \frac{\partial^{2} \bar{u}(p, q, s)}{\partial q \partial s}+p^{2} q s^{2} \frac{\partial^{2} \bar{u}(p, q, s)}{\partial p \partial s} \\
& +p^{2} q^{2} s \frac{\partial^{2} \bar{u}(p, q, s)}{\partial p \partial q}+p^{2} q s \frac{\partial \bar{u}(p, q, s)}{\partial p}+p q^{2} s \frac{\partial \bar{u}(p, q, s)}{\partial q} \\
+ & p q s^{2} \frac{\partial \bar{u}(p, q, s)}{\partial s}+p q s \bar{u}(p, q, s) .
\end{aligned}
$$

Hence, the result (6) is proved.

Theorem 4.3. For $0<\alpha, \beta, \gamma \leq 1$, the Conformable fractional triple Sumudu transformation of $\frac{\partial^{\alpha} \varphi}{\partial x^{\alpha}}, \frac{\partial^{\beta} \varphi}{\partial y^{\beta}}, \frac{\partial^{\gamma} \varphi}{\partial t^{\gamma}}$ can be represented as follows:

1. $S_{x}^{\alpha} S_{y}^{\beta} S_{t}^{\gamma}\left(\frac{\partial^{\alpha} \varphi}{\partial x^{\alpha}}\right)=p^{-1}[\bar{\varphi}(p, q, s)-\bar{\varphi}(0, q, s)]$.

2. $S_{x}^{\alpha} S_{y}^{\beta} S_{t}^{\gamma}\left(\frac{\partial^{\beta} \varphi}{\partial y^{\beta}}\right)=q^{-1}[\bar{\varphi}(p, q, s)-\bar{\varphi}(p, 0, s)]$.

3. $S_{x}^{\alpha} S_{y}^{\beta} S_{t}^{\gamma}\left(\frac{\partial^{\gamma} \varphi}{\partial t^{\gamma}}\right)=s^{-1}[\bar{\varphi}(p, q, s)-\bar{\varphi}(p, q, 0)]$.

Proof. (1) By applying the definition of CFTST, we get,

$$
\begin{aligned}
& S_{x}^{\alpha} S_{y}^{\beta} S_{t}^{\gamma}\left(\frac{\partial^{\alpha} \varphi}{\partial x^{\alpha}}\right)=\frac{1}{p q S} \int_{0}^{\infty} \int_{0}^{\infty} \int_{0}^{\infty} e^{-\left(\frac{x^{\alpha}}{\alpha p}\right)-\left(\frac{y^{\beta}}{\beta q}\right)-\left(\frac{t \gamma}{\gamma s}\right)} \frac{\partial^{\alpha} \varphi}{\partial x^{\alpha}} x^{\alpha-1} y^{\beta-1} t^{\gamma-1} \mathrm{~d} x \mathrm{~d} y \mathrm{~d} t \\
= & \int_{0}^{\infty} \int_{0}^{\infty} e^{-q\left(\frac{y^{\beta}}{\beta}\right)-s\left(\frac{t \gamma}{\gamma}\right)}\left(\int_{0}^{\infty} e^{-\left(\frac{x^{\alpha}}{\alpha p}\right)} \frac{\partial^{\alpha} \varphi}{\partial x^{\alpha}} x^{\alpha-1} \mathrm{~d} x\right) y^{\beta-1} t^{\gamma-1} \mathrm{~d} y \mathrm{~d} t .
\end{aligned}
$$

Since we have the result, $\frac{\partial^{\alpha} \varphi}{\partial x^{\alpha}}=x^{1-\alpha} \frac{\partial \varphi}{\partial x}$, and simplifying Eq. (4.14), we have

$$
=\frac{1}{p q s} \int_{0}^{\infty} \int_{0}^{\infty} e^{-\left(\frac{y^{\beta}}{\beta q}\right)-\left(\frac{t^{\gamma}}{\gamma s}\right)}\left(\int_{0}^{\infty} e^{-\left(\frac{x^{\alpha}}{\alpha p}\right)} \frac{\partial \varphi}{\partial x} \mathrm{~d} x\right) y^{\beta-1} t^{\gamma-1} \mathrm{~d} y \mathrm{~d} t .
$$

Using the integration by part rule, Eq. (4.15) reduces to,

$$
S_{x}^{\alpha} S_{y}^{\beta} S_{t}^{\gamma}\left(\frac{\partial^{\alpha} \varphi}{\partial x^{\alpha}}\right)=\frac{1}{p}[\bar{\varphi}(p, q, s)-\bar{\varphi}(0, q, s)],
$$

which complete the proof (1). Similarly, result (2) and (3) can be proved. 
The following lemma also can be proved by similar way.

Lemma 4.1. If $S_{x}^{\alpha} S_{y}^{\beta} S_{t}^{\gamma}\left(\varphi\left(\frac{x^{\alpha}}{\alpha}, \frac{y^{\beta}}{\beta}, \frac{t^{\gamma}}{\gamma}\right)\right)=\bar{\varphi}(p, q, s)$, the conformable fractional triple Sumudu transform of the $\frac{\partial^{2 \alpha} \varphi}{\partial x^{2 \alpha}}, \frac{\partial^{2 \alpha} \varphi}{\partial y^{2 \alpha}}, \frac{\partial^{2 \alpha} \varphi}{\partial t^{2 \alpha}}, \frac{\partial^{3 \alpha} \varphi}{\partial x^{3 \alpha}}$ is given by: For $0<\alpha, \beta, \gamma \leq 1$,

(i) $S_{x}^{\alpha} S_{y}^{\beta} S_{t}^{\gamma}\left(\frac{\partial^{2 \alpha} \varphi}{\partial x^{2 \alpha}}\right)=p^{-2} \bar{\varphi}(p, q, s)-p^{-2} \bar{\varphi}(0, q, s)-p^{-1} \bar{\varphi}_{x}(0, q, s)$.

(ii) $S_{x}^{\alpha} S_{y}^{\beta} S_{t}^{\gamma}\left(\frac{\partial^{2 \beta} \varphi}{\partial y^{2 \beta}}\right)=q^{-2} \bar{\varphi}(p, q, s)-q^{-2} \bar{\varphi}(p, 0, s)-q^{-1} \bar{\varphi}_{y}(p, 0, s)$.

(iii) $S_{x}^{\alpha} S_{y}^{\beta} S_{t}^{\gamma}\left(\frac{\partial^{2 \gamma} \varphi}{\partial t^{2 \gamma}}\right)=s^{-2} \bar{\varphi}(p, q, s)-s^{-2} \bar{\varphi}(p, q, 0)-s^{-1} \bar{\varphi}_{t}(p, q, 0)$.

(iv) $S_{x}^{\alpha} S_{y}^{\beta} S_{t}^{\gamma}\left(\frac{\partial^{3 \alpha} \varphi}{\partial x^{3 \alpha}}\right)=p^{-3} \bar{\varphi}(p, q, s)-p^{-3} \bar{\varphi}(0, q, s)-p^{-2} \bar{\varphi}_{x}(0, q, s)-p^{-1} \bar{\varphi}_{x x}(0, q, s)$.

Proof. The proof can be followed from Theorem 4.3.

In general, the above lemma can be extended as follows and it can be verified by mathematical induction:

1. $S_{x}^{\alpha} S_{y}^{\beta} S_{t}^{\gamma}\left(\frac{\partial^{\alpha+\beta+\gamma} \varphi}{\partial x^{\alpha} \partial y^{\beta} \partial t^{\gamma}}\right)=p^{-1} q^{-1} s^{-1} \bar{\varphi}(p, q, s)-p^{-1} \bar{\varphi}(p, 0,0)-q^{-1} \bar{\varphi}(0, q, 0)-s^{-1} \bar{\varphi}(0,0, s)$ $-p^{-1} q^{-1} \bar{\varphi}(p, q, 0)-p^{-1} s^{-1} \bar{\varphi}(p, 0, s)-q^{-1} \mathcal{S}^{-1} \bar{\varphi}(0, q, s)-\bar{\varphi}(0,0,0)$.

2. $S_{x}^{\alpha} S_{y}^{\beta} S_{t}^{\gamma}\left(\frac{\partial^{\alpha l} \varphi}{\partial x^{\alpha l}}\right)=p^{-l}\left[\bar{\varphi}(p, q, s)-\sum_{k=0}^{l-1} p^{k} S_{y}^{\beta} S_{t}^{\gamma}\left(\frac{\partial^{\alpha k}}{\partial x^{\alpha k}} \varphi(0, y, t)\right)\right]$.

3. $S_{x}^{\alpha} S_{y}^{\beta} S_{t}^{\gamma}\left(\frac{\partial^{\beta m} \varphi}{\partial y^{\beta m}}\right)=q^{-m}\left[\bar{\varphi}(p, q, s)-\sum_{k=0}^{m-1} q^{k} S_{x}^{\alpha} S_{t}^{\gamma}\left(\frac{\partial^{\beta k}}{\partial y^{\beta k}} \varphi(x, 0, t)\right)\right]$.

4. $S_{x}^{\alpha} S_{y}^{\beta} S_{t}^{\gamma}\left(\frac{\partial^{\gamma n} \varphi}{\partial t^{\gamma n}}\right)=s^{-n}\left[\bar{\varphi}(p, q, s)-\sum_{k=0}^{n-1} s^{k} S_{x}^{\alpha} S_{y}^{\beta}(\varphi(x, y, 0))\right]$.

The following theorem gives the conformable triple Sumudu transform of the fractional partial derivatives $\frac{x^{\alpha}}{\alpha} \frac{\partial^{\gamma} \varphi}{\partial t^{\gamma}}$ and $\frac{x^{\alpha}}{\alpha} \frac{\partial^{2 \gamma} \varphi}{\partial t^{2 \gamma}}$.

Theorem 4.4. Let $S_{x}^{\alpha} S_{y}^{\beta} S_{t}^{\gamma}\left(\varphi\left(\frac{x^{\alpha}}{\alpha}, \frac{y^{\beta}}{\beta}, \frac{t^{\gamma}}{\gamma}\right)\right)=\bar{\varphi}(p, q, s)$. If the conformable triple Sumudu transform of the function $\frac{x^{\alpha}}{\alpha} \frac{\partial^{\gamma} \varphi}{\partial t^{\gamma}}$ and $\frac{x^{\alpha}}{\alpha} \frac{\partial^{2 \gamma} \varphi}{\partial t^{2 \gamma}}$ are given $b y$ :

For $0<\alpha, \beta, \gamma \leq 1$.

1. $S_{x}^{\alpha} S_{y}^{\beta} S_{t}^{\gamma}\left(\frac{x^{\alpha}}{\alpha} \frac{\partial^{\gamma} \varphi}{\partial t^{\gamma}}\right)=\frac{p}{S}\left(\frac{\mathrm{d}}{\mathrm{d} p}(p \bar{\varphi}(p, q, s))-\frac{\mathrm{d}}{\mathrm{d} p}(p \bar{\varphi}(p, q, 0))\right)$. 
2. $S_{x}^{\alpha} S_{y}^{\beta} S_{t}^{\gamma}\left(\frac{x^{\alpha}}{\alpha} \frac{\partial^{2 \gamma} \varphi}{\partial t^{2 \gamma}}\right)=\frac{p}{s^{2}}\left(\frac{\mathrm{d}}{\mathrm{d} p}(p \bar{\varphi}(p, q, s))-\frac{\mathrm{d}}{\mathrm{d} p}(p \bar{\varphi}(p, q, 0))\right)$

$$
-\frac{p}{S} \frac{\mathrm{d}}{\mathrm{d} p}\left(p S_{x}^{\alpha} S_{y}^{\beta} S_{t}^{\gamma}\left(\frac{\partial^{\gamma} \varphi\left(\frac{x^{\alpha}}{\alpha}, \frac{y^{\beta}}{\beta}, 0\right)}{\partial t^{\gamma}}\right)\right) .
$$

Proof. By using the definition of triple Sumudu transform, we have

$$
S_{x}^{\alpha} S_{y}^{\beta} S_{t}^{\gamma}\left(\frac{\partial^{\gamma} \varphi}{\partial t \gamma}\right)=\frac{1}{p q S} \int_{0}^{\infty} \int_{0}^{\infty} \int_{0}^{\infty} e^{-\left(\frac{x^{\alpha}}{p \alpha}\right)-\left(\frac{y^{\beta}}{q \beta}\right)-\left(\frac{t \gamma}{s \gamma}\right)} \frac{\partial^{\gamma} \varphi}{\partial t \gamma} x^{\alpha-1} y^{\beta-1} t^{\gamma-1} \mathrm{~d} x \mathrm{~d} y \mathrm{~d} t .
$$

Now differentiating with respect to $p$, we get

$$
\begin{aligned}
& \frac{\mathrm{d}}{\mathrm{d} p}\left(S_{x}^{\alpha} S_{y}^{\beta} S_{t}^{\gamma}\left(\frac{\partial^{\gamma} \varphi}{\partial t \gamma}\right)\right) \\
= & \frac{\mathrm{d}}{\mathrm{d} p}\left(\frac{1}{p q s} \int_{0}^{\infty} \int_{0}^{\infty} \int_{0}^{\infty}\left(\begin{array}{c}
e^{-\left(\frac{x^{\alpha}}{p \alpha}\right)-\left(\frac{y^{\beta}}{q \beta}\right)-\left(\frac{t \gamma}{s \gamma}\right)} \\
\frac{\partial^{\gamma} \varphi}{\partial t \gamma} x^{\alpha-1} y^{\beta-1} t^{\gamma-1}
\end{array}\right) \mathrm{d} x \mathrm{~d} y \mathrm{~d} t\right) \\
= & \frac{1}{q S} \int_{0}^{\infty} \int_{0}^{\infty} e^{-\left(\frac{y^{\beta}}{q \beta}\right)-\left(\frac{t^{\gamma}}{s \gamma}\right)} \frac{\partial^{\gamma} \varphi}{\partial t \gamma}\left(\int_{0}^{\infty} \frac{\partial}{\partial p}\left(\frac{1}{p} e^{-\left(\frac{x^{\alpha}}{p \alpha}\right)}\right) x^{\alpha-1} d x\right) y^{\beta-1} t^{\gamma-1} \mathrm{~d} y \mathrm{~d} t \\
= & \frac{1}{q S} \int_{0}^{\infty} \int_{0}^{\infty} e^{-\left(\frac{y^{\beta}}{q \beta}\right)-\left(\frac{t \gamma}{s \gamma}\right)} \frac{\partial^{\gamma} \varphi}{\partial t \gamma}\left(\int_{0}^{\infty}\left(\frac{1}{p^{3}} \frac{x^{\alpha}}{\alpha}-\frac{1}{p^{2}}\right) e^{-\left(\frac{x^{\alpha}}{p \alpha}\right)} x^{\alpha-1} d x\right) y^{\beta-1} t^{\gamma-1} \mathrm{~d} y \mathrm{~d} t \\
= & \frac{1}{p^{3} q S} \int_{0}^{\infty} \int_{0}^{\infty} \int_{0}^{\infty} e^{-\left(\frac{x^{\alpha}}{p \alpha}\right)-\left(\frac{y^{\beta}}{q \beta}\right)-\left(\frac{t^{\gamma}}{s \gamma}\right)}\left(\frac{x^{\alpha}}{\alpha} \frac{\partial^{\gamma} \varphi}{\partial t \gamma}\right) x^{\alpha-1} y^{\beta-1} t^{\gamma-1} \mathrm{~d} x \mathrm{~d} y \mathrm{~d} t \\
& \quad-\frac{1}{p^{2} q S} \int_{0}^{\infty} \int_{0}^{\infty} \int_{0}^{\infty} e^{-\left(\frac{x^{\alpha}}{p \alpha}\right)-\left(\frac{y^{\beta}}{q \beta}\right)-\left(\frac{t^{\gamma}}{s \gamma}\right)}\left(\frac{\partial^{\gamma} \varphi}{\partial t^{\gamma}}\right) x^{\alpha-1} y^{\beta-1} t^{\gamma-1} \mathrm{~d} x \mathrm{~d} y \mathrm{~d} t .
\end{aligned}
$$

Therefore,

$$
S_{x}^{\alpha} S_{y}^{\beta} S_{t}^{\gamma}\left(\frac{x^{\alpha}}{\alpha} \frac{\partial^{\gamma} \varphi}{\partial t^{\gamma}}\right)=p^{2} \frac{\mathrm{d}}{\mathrm{d} p}\left(S_{x}^{\alpha} S_{y}^{\beta} S_{t}^{\gamma}\left(\frac{\partial^{\gamma} \varphi}{\partial t^{\gamma}}\right)\right)+p S_{x}^{\alpha} S_{y}^{\beta} S_{t}^{\gamma}\left(\frac{\partial^{\gamma} \varphi}{\partial t^{\gamma}}\right)
$$

We use Theorem 4.3 and simplifying, we get

$$
\begin{aligned}
& S_{x}^{\alpha} S_{y}^{\beta} S_{t}^{\gamma}\left(\frac{x^{\alpha}}{\alpha} \frac{\partial^{\gamma} \varphi}{\partial t^{\gamma}}\right)=p^{2} \frac{\mathrm{d}}{\mathrm{d} p}\left(\left(s^{-1}[\bar{\varphi}(p, q, s)-\bar{\varphi}(p, q, 0)]\right)\right)+p\left(s^{-1}\left[\begin{array}{c}
\bar{\varphi}(p, q, s) \\
-\bar{\varphi}(p, q, 0)
\end{array}\right]\right) . \\
& S_{x}^{\alpha} S_{y}^{\beta} S_{t}^{\gamma}\left(\frac{x^{\alpha}}{\alpha} \frac{\partial^{\gamma} \varphi}{\partial t^{\gamma}}\right)=\frac{p^{2}}{\mathrm{~s}} \frac{\mathrm{d}}{\mathrm{d} p}(\bar{\varphi}(p, q, s))-\frac{p^{2}}{s} \frac{\mathrm{d}}{\mathrm{d} p}(\bar{\varphi}(p, q, 0))+\frac{p}{s}(\bar{\varphi}(p, q, s)-\bar{\varphi}(p, q, 0)) . \\
& S_{x}^{\alpha} S_{y}^{\beta} S_{t}^{\gamma}\left(\frac{x^{\alpha}}{\alpha} \frac{\partial^{\gamma} \varphi}{\partial t^{\gamma}}\right)=\frac{p}{s}\left(\frac{d}{d p}(p \bar{\varphi}(p, q, s))-\frac{\mathrm{d}}{\mathrm{d} p}(p \bar{\varphi}(p, q, 0))\right) .
\end{aligned}
$$


Hence, result- 1 is proved. In a similar manner, one can prove result-2.

\section{The fractional conformable triple Sumudu and Laplace transform and Adomian decomposition method}

In this section we propose conformable triple Sumudu Adomian decomposition method. Similarly, conformable triple Laplace Adomian decomposition method can be followed. Consider nonlinear non homogeneous partial fractional differential equation,

$$
\frac{\partial^{\gamma n}}{\partial t^{\gamma n}}\left(u\left(\frac{x^{\alpha}}{\alpha}, \frac{y^{\beta}}{\beta}, \frac{t^{\gamma}}{\gamma}\right)\right)+R u\left(\frac{x^{\alpha}}{\alpha}, \frac{y^{\beta}}{\beta}, \frac{t^{\gamma}}{\gamma}\right)+N u\left(\frac{x^{\alpha}}{\alpha}, \frac{y^{\beta}}{\beta}, \frac{t^{\gamma}}{\gamma}\right)=g\left(\frac{x^{\alpha}}{\alpha}, \frac{y^{\beta}}{\beta}, \frac{t^{\gamma}}{\gamma}\right),
$$

where $m=1,2,3, \ldots$ and $\gamma \in(0,1]$ with the initial conditions,

$$
\frac{\partial^{\gamma n-1}}{\partial t^{\prime n-1}}\left(u\left(\frac{x^{\alpha}}{\alpha}, \frac{y^{\beta}}{\beta}, 0\right)\right)=f_{\gamma n-1}\left(\frac{x^{\alpha}}{\alpha}, \frac{y^{\beta}}{\beta}, 0\right),
$$

where $\mathrm{R}$ is the linear differential operator, $\mathrm{N}$ addresses the non-Linear partial fractional operator and $g\left(\frac{x^{\alpha}}{\alpha}, \frac{y^{\beta}}{\beta}, \frac{t \gamma}{\gamma}\right)$, is the source term.

To solve equation (5.1) follow the following steps:

Step 1: Take fractional conformable triple Sumudu Transform to the Eq. (5.1)on both sides, and applying standard result. we have,

$$
\begin{aligned}
& S_{x}^{\alpha} S_{y}^{\beta} S_{t}^{\gamma} \frac{\partial^{\gamma n}}{\partial t^{\gamma n}}\left(u\left(\frac{x^{\alpha}}{\alpha}, \frac{y^{\beta}}{\beta}, \frac{t^{\gamma}}{\gamma}\right)\right)+S_{x}^{\alpha} S_{y}^{\beta} S_{t}^{\gamma}\left(R u\left(\frac{x^{\alpha}}{\alpha}, \frac{y^{\beta}}{\beta}, \frac{t^{\gamma}}{\gamma}\right)\right)+ \\
& S_{x}^{\alpha} S_{y}^{\beta} S_{t}^{\gamma}\left(N u\left(\frac{x^{\alpha}}{\alpha}, \frac{y^{\beta}}{\beta}, \frac{t^{\gamma}}{\gamma}\right)\right)=S_{x}^{\alpha} S_{y}^{\beta} S_{t}^{\gamma}\left(g\left(\frac{x^{\alpha}}{\alpha}, \frac{y^{\beta}}{\beta}, \frac{t^{\gamma}}{\gamma}\right)\right) . \\
& \therefore s^{-n}\left[\bar{u}(p, q, s)-\sum_{k=0}^{n-1} s^{k} S_{x}^{\alpha} S_{y}^{\beta}(u(x, y, 0))\right]+S_{x}^{\alpha} S_{y}^{\beta} S_{t}^{\gamma}\left(R u\left(\frac{x^{\alpha}}{\alpha}, \frac{y^{\beta}}{\beta}, \frac{t^{\gamma}}{\gamma}\right)\right)+ \\
& S_{x}^{\alpha} S_{y}^{\beta} S_{t}^{\gamma}\left(N u\left(\frac{x^{\alpha}}{\alpha}, \frac{y^{\beta}}{\beta}, \frac{t^{\gamma}}{\gamma}\right)\right)=S_{x}^{\alpha} S_{y}^{\beta} S_{t}^{\gamma}\left(g\left(\frac{x^{\alpha}}{\alpha}, \frac{y^{\beta}}{\beta}, \frac{t^{\gamma}}{\gamma}\right)\right) .
\end{aligned}
$$

Step 2: Using the derived result and applying the fractional conformable inverse triple Sumudu transform. we get,

$$
u(x, y, t)=G(x, y, t)-S_{p}^{-1} S_{q}^{-1} S_{s}^{-1}\left\{s^{n}\left[S_{x}^{\alpha} S_{y}^{\beta} S_{t}^{\gamma}\left(\begin{array}{c}
R u\left(\frac{x^{\alpha}}{\alpha}, \frac{y^{\beta}}{\beta}, \frac{t^{\gamma}}{\gamma}\right) \\
+N u\left(\frac{x^{\alpha}}{\alpha}, \frac{y^{\beta}}{\beta}, \frac{t^{\gamma}}{\gamma}\right)
\end{array}\right)\right]\right\},
$$


where $G(x, y, t)$ represents the term coming from the source term and prescribed initial conditions.

Step 3: Apply the Adomian decomposition method, let the solution of the Eq. (5.1) series,

$$
u\left(\frac{x^{\alpha}}{\alpha}, \frac{y^{\beta}}{\beta}, \frac{t^{\gamma}}{\gamma}\right)=\sum_{n=0}^{\infty} u_{n}\left(\frac{x^{\alpha}}{\alpha}, \frac{y^{\beta}}{\beta}, \frac{t^{\gamma}}{\gamma}\right),
$$

and the nonlinear term can be decomposed as

$$
N u\left(\frac{x^{\alpha}}{\alpha}, \frac{y^{\beta}}{\beta}, \frac{t^{\gamma}}{\gamma}\right)=\sum_{n=0}^{\infty} A_{n}
$$

where $A_{n}$ is called Adomian Polynomials of $u_{1}, u_{2}, u_{3}, . ., u_{n}$ and it can be calculated by the following formula:

$$
A_{n}=\frac{1}{n !}\left[\frac{\partial^{n}}{\partial \sigma^{n}}\left(N \sum_{i=1}^{\infty} \sigma^{i} u_{i}\right)\right]_{\sigma=0}
$$

where $n=0,1,2,3,4, \ldots \ldots \ldots$

Substituting Eqs. (5.5) and (5.6) in the Eq. (5.4), we have

$$
\begin{aligned}
& \sum_{n=0}^{\infty} u_{n}\left(\frac{x^{\alpha}}{\alpha}, \frac{y^{\beta}}{\beta}, \frac{t^{\gamma}}{\gamma}\right) \\
= & G(x, y, t)-S_{p}^{-1} S_{q}^{-1} S_{s}^{-1}\left\{s^{n}\left[\begin{array}{c}
S_{x}^{\alpha} S_{y}^{\beta} S_{t}^{\gamma}\left(R \sum_{n=0}^{\infty} u_{n}\left(\frac{x^{\alpha}}{\alpha}, \frac{y^{\beta}}{\beta}, \frac{t^{\gamma}}{\gamma}\right)\right) \\
+S_{x}^{\alpha} S_{y}^{\beta} S_{t}^{\gamma}\left(N \sum_{n=0}^{\infty} A_{n}\right)
\end{array}\right]\right\} .
\end{aligned}
$$

Step 4: The recursive relation is given by,

$$
\begin{aligned}
& u_{0}\left(\frac{x^{\alpha}}{\alpha}, \frac{y^{\beta}}{\beta}, \frac{t^{\gamma}}{\gamma}\right)=G(x, y, t) . \\
& u_{m+1}\left(\frac{x^{\alpha}}{\alpha}, \frac{y^{\beta}}{\beta}, \frac{t^{\gamma}}{\gamma}\right)=-S_{p}^{-1} S_{q}^{-1} S_{s}^{-1}\left\{s^{n}\left[S_{x}^{\alpha} S_{y}^{\beta} S_{t}^{\gamma}\left(R u_{n}\left(\frac{x^{\alpha}}{\alpha}, \frac{y^{\beta}}{\beta}, \frac{t^{\gamma}}{\gamma}\right)+N A_{n}\right)\right]\right\},
\end{aligned}
$$

where $n=1,2,3, \ldots$ and $m=0,1,2,3, \ldots$

At the end, we can approximate the dynamic analytical solution, as

$$
u\left(\frac{x^{\alpha}}{\alpha}, \frac{y^{\beta}}{\beta}, \frac{t^{\gamma}}{\gamma}\right)=\sum_{m=0}^{\infty} u_{m}\left(\frac{x^{\alpha}}{\alpha}, \frac{y^{\beta}}{\beta}, \frac{t^{\gamma}}{\gamma}\right) .
$$




\section{Applications}

In this section, we do experiment and analyzed by both the proposed method.

Example 6.1: Solve by Fractional conformable Sumudu transform decomposition method. Consider dynamic nonlinear non homogeneous partial fractional differential equation: For $\alpha, \beta, \gamma \in(0,1], x, y, t \in[0, \infty)$.

$$
\frac{\partial^{\beta} u}{\partial y^{\beta}} \frac{\partial^{\gamma} u}{\partial t^{\gamma}}-\frac{\partial^{2 \alpha} u}{\partial x^{2 \alpha}}=u(x, y, t)
$$

with the initial conditions,

$$
u(0, y, t)=y t, \quad u_{x}(0, y, t)=-1 .
$$

Solution: Applying the conformable fractional triple Sumudu transform to both sides of Eq. (6.1)

$$
S_{x}^{\alpha} S_{y}^{\beta} S_{t}^{\gamma}\left(\frac{\partial^{2 \alpha} u}{\partial x^{2 \alpha}}\right)=S_{x}^{\alpha} S_{y}^{\beta} S_{t}^{\gamma}\left(\frac{\partial^{\beta} u}{\partial y^{\beta}} \frac{\partial^{\gamma} u}{\partial t^{\gamma}}-u(x, y, t)\right) .
$$

Recalling Lemma-4.1 (i), we obtain,

$$
\bar{\varphi}(p, q, s)=\bar{\varphi}(0, q, s)+p \bar{\varphi}_{x}(0, q, s)+p^{2} S_{x}^{\alpha} S_{y}^{\beta} S_{t}^{\gamma}\left(\frac{\partial^{\beta} u}{\partial y^{\beta}} \frac{\partial^{\gamma} u}{\partial t^{\gamma}}-u(x, y, t)\right) .
$$

Using initial condition Eq. (6.2) and the inverse triple Sumudu transform, we obtain

$$
\begin{aligned}
& u(x, y, t)=S_{p}^{-1} S_{q}^{-1} S_{s}^{-1}(q s-p)+S_{p}^{-1} S_{q}^{-1} S_{s}^{-1}\left(p^{2} S_{x}^{\alpha} S_{y}^{\beta} S_{t}^{\gamma}\left(\frac{\partial^{\beta} u}{\partial y^{\beta}} \frac{\partial^{\gamma} u}{\partial t^{\gamma}}-u(x, y, t)\right)\right) . \\
& u(x, y, t)=y t-x+S_{p}^{-1} S_{q}^{-1} S_{s}^{-1}\left(p^{2} S_{x}^{\alpha} S_{y}^{\beta} S_{t}^{\gamma}\left(\frac{\partial^{\beta} u}{\partial y^{\beta}} \frac{\partial^{\gamma} u}{\partial t^{\gamma}}-u(x, y, t)\right)\right) .
\end{aligned}
$$

Applying the proposed method, we get the recursive formula,

$$
\begin{aligned}
& u_{0}=y t-x \\
& u_{n+1}=S_{p}^{-1} S_{q}^{-1} S_{s}^{-1}\left(p^{2} S_{x}^{\alpha} S_{y}^{\beta} S_{t}^{\gamma}\left(\frac{\partial^{\beta} u_{n}}{\partial y^{\beta}} \frac{\partial^{\gamma} u_{n}}{\partial t^{\gamma}}-u_{n}\right)\right) \\
& \quad=S_{p}^{-1} S_{q}^{-1} S_{s}^{-1}\left(p^{2} S_{x}^{\alpha} S_{y}^{\beta} S_{t}^{\gamma}\left(A_{n}-u_{n}\right)\right)
\end{aligned}
$$

where $A_{n}$ is the Adomian polynomial to decompose the nonlinear terms by using the relation;

$$
A_{n}=\frac{1}{n !}\left[\frac{\partial^{n}}{\partial \sigma^{n}}\left(N \sum_{i=1}^{\infty} \sigma^{i} u_{i}\right)\right]_{\sigma=0},
$$

where $n=0,1,2,3,4, \ldots \ldots \ldots$ 
Let the nonlinear term can be represented as,

$$
f(u)=\frac{\partial^{\beta} u}{\partial y^{\beta}} \frac{\partial^{\gamma} u}{\partial t^{\gamma}}
$$

Eqs. (6.6)-(6.8) gives,

$$
\begin{aligned}
& u_{1}=\frac{x^{2}}{2 !} y^{2-\beta} t^{2-\gamma}-\frac{x^{2}}{2 !} y t+\frac{x^{3}}{3 !} . \\
& u_{2}=(4-\gamma-\beta) \frac{x^{4}}{4 !} y^{3-2 \beta} t^{3-2 \gamma}-\frac{x^{4}}{8} y^{2-\beta} t^{2-\gamma}+\frac{x^{4}}{4 !} y t-\frac{x^{5}}{5 !} .
\end{aligned}
$$

And so on...

The approximate series solution is,

$$
u(x, y, t)=(y t-x)+\left(\frac{x^{2}}{2} y^{2-\beta} t^{2-\gamma}-\frac{x^{2}}{2} y t+\frac{x^{3}}{3 !}\right)+\left(\begin{array}{c}
(4-\gamma-\beta) \frac{x^{4}}{4 !} y^{3-2 \beta} t^{3-2 \gamma} \\
-\frac{x^{4}}{8} y^{2-\beta} t^{2-\gamma} \\
+\frac{x^{4}}{4 !} y t-\frac{x^{5}}{5 !}
\end{array}\right)+\ldots
$$

for $\alpha, \beta, \gamma \in(0,1], x, y, t \in[0, \infty)$.

Note that for $\alpha, \beta, \gamma=1$, the solution of Eq. (6.1) from Eq. (6.10), as

$$
u(x, y, t)=y t-\left(x-\frac{x^{3}}{3 !}+\frac{x^{5}}{5 !}+\ldots\right)=y t-\sin x,
$$

which is exact solution of integer order of partial differential equation.

Example 6.2: Solve by fractional conformable Laplace decomposition method. Consider nonlinear non homogeneous partial fractional differential equation, for $\alpha, \beta, \gamma \in$ $(0,1], x, y, t \in[0, \infty)$.

$$
\frac{\partial^{\beta} u}{\partial y^{\beta}} \frac{\partial^{\gamma} u}{\partial t^{\gamma}}-\frac{\partial^{2 \alpha} u}{\partial x^{2 \alpha}}=u(x, y, t)
$$

with initial conditions,

$$
u(0, y, t)=y t, \quad u_{x}(0, y, t)=-1
$$

Solution: Rewriting, Eq. (6.11) as:

$$
\frac{\partial^{2 \alpha} u}{\partial x^{2 \alpha}}=\frac{\partial^{\beta} u}{\partial y^{\beta}} \frac{\partial^{\gamma} u}{\partial t^{\gamma}}-u(x, y, t)
$$

Imposing the fractional conformable triple Laplace transform to both sides,

$$
L_{x}^{\alpha} L_{y}^{\beta} L_{t}^{\gamma}\left(\frac{\partial^{2 \alpha} u}{\partial x^{2 \alpha}}\right)=L_{x}^{\alpha} L_{y}^{\beta} L_{t}^{\gamma}\left(\frac{\partial^{\beta} u}{\partial y^{\beta}} \frac{\partial^{\gamma} u}{\partial t^{\gamma}}-u(x, y, t)\right)
$$


Recalling Theorem $3.4(4): L_{x}^{\alpha} L_{y}^{\beta} L_{t}^{\gamma}\left(\frac{\partial^{2 \alpha}}{\partial x^{2 \alpha}}(u(x, y, t))\right)=p^{2} U(p, q, s)-p U(0, q, s)-U_{x}(0, q, s)$, Eq. (6.14) becomes,

$$
p^{2} U(p, q, s)=p U(0, q, s)+U_{x}(0, q, s)+L_{x}^{\alpha} L_{y}^{\beta} L_{t}^{\gamma}\left(\frac{\partial^{\beta} u}{\partial y^{\beta}} \frac{\partial^{\gamma} u}{\partial t^{\gamma}}-u(x, y, t)\right) .
$$

Using initial condition (6.12) and taking the inverse triple Laplace transform on Eq. (6.15), we obtain:

$$
\begin{aligned}
& u(x, y, t)=\mathrm{L}_{\mathrm{p}}^{-1} \mathrm{~L}_{\mathrm{q}}^{-1} \mathrm{~L}_{\mathrm{s}}^{-1}\left(\frac{1}{p q^{2} s^{2}}-\frac{1}{p^{2} q s}\right)+\mathrm{L}_{\mathrm{p}}^{-1} \mathrm{~L}_{\mathrm{q}}^{-1} \mathrm{~L}_{\mathrm{s}}^{-1}\left(\frac{1}{p^{2}} L_{x}^{\alpha} L_{y}^{\beta} L_{t}^{\gamma}\left(\begin{array}{c}
\frac{\partial^{\beta} u}{\partial y^{\beta}} \frac{\partial^{\gamma} u}{\partial t^{\gamma}} \\
-u(x, y, t)
\end{array}\right)\right) \\
& u(x, y, t)=y t-x+\mathrm{L}_{\mathrm{p}}^{-1} \mathrm{~L}_{\mathrm{q}}^{-1} \mathrm{~L}_{\mathrm{s}}^{-1}\left(\frac{1}{p^{2}} L_{x}^{\alpha} L_{y}^{\beta} L_{t}^{\gamma}\left(\frac{\partial^{\beta} u}{\partial y^{\beta}} \frac{\partial^{\gamma} u}{\partial t^{\gamma}}-u(x, y, t)\right)\right) .
\end{aligned}
$$

Applying the proposed method, let

$$
u_{0}=y t-x
$$

And the recursive relation is,

$$
\begin{aligned}
u_{n+1} & =\mathrm{L}_{\mathrm{p}}^{-1} \mathrm{~L}_{\mathrm{q}}^{-1} \mathrm{~L}_{\mathrm{s}}^{-1}\left(\frac{1}{p^{2}} L_{x}^{\alpha} L_{y}^{\beta} L_{t}^{\gamma}\left(\frac{\partial^{\beta} u_{n}}{\partial y^{\beta}} \frac{\partial^{\gamma} u_{n}}{\partial t^{\gamma}}-u_{n}\right)\right) \\
& =\mathrm{L}_{\mathrm{p}}^{-1} \mathrm{~L}_{\mathrm{q}}^{-1} \mathrm{~L}_{\mathrm{s}}^{-1}\left(\frac{1}{p^{2}} L_{x}^{\alpha} L_{y}^{\beta} L_{t}^{\gamma}\left(A_{n}-u_{n}\right)\right),
\end{aligned}
$$

where $A_{n}$ is the Adomian polynomial to decompose the nonlinear terms by using the relation;

$$
A_{n}=\frac{1}{n !}\left[\frac{\partial^{n}}{\partial \sigma^{n}}\left(N \sum_{i=1}^{\infty} \sigma^{i} u_{i}\right)\right]_{\sigma=0}
$$

where $n=0,1,2,3,4$,

Let the nonlinear term be represented as,

$$
f(u)=\frac{\partial^{\beta} u}{\partial y^{\beta}} \frac{\partial^{\gamma} u}{\partial t^{\gamma}}
$$

Note that, in Adomian relation, the linear term can be considered, in place of nonlinear. So, you may take $f(u)=u$ and it leads to the same answer. So, this method is also valid for linear partial fractional differential equation.

For $n=0$,

$$
A_{0}=f\left(u_{0}\right)=\frac{\partial^{\beta} u_{0}}{\partial y^{\beta}} \frac{\partial^{\gamma} u_{0}}{\partial t^{\gamma}}=\left(y^{1-\beta} t\right)\left(y t^{1-\gamma}\right)=y^{2-\beta} t^{2-\gamma}
$$


Therefore,

$$
\begin{aligned}
u_{1} & =\mathrm{L}_{\mathrm{p}}^{-1} \mathrm{~L}_{\mathrm{q}}^{-1} \mathrm{~L}_{\mathrm{s}}^{-1}\left(\frac{1}{p^{2}} L_{x}^{\alpha} L_{y}^{\beta} L_{t}^{\gamma}\left(y^{2-\beta} t^{2-\gamma}-(y t-x)\right)\right) \\
& =\frac{x^{2}}{2} y^{2-\beta} t^{2-\gamma}-\frac{x^{2}}{2} y t+\frac{x^{3}}{3 !} .
\end{aligned}
$$

For $n=1$,

$$
\begin{aligned}
A_{1} & =\left.\frac{\partial}{\partial \sigma}\left[f\left(u_{0}+\sigma u_{1}\right)\right]\right|_{\sigma=0} \\
& =\left.\frac{\partial}{\partial \sigma}\left(\frac{\partial^{\beta}\left(u_{0}+\sigma u_{1}\right)}{\partial y^{\beta}} \frac{\partial^{\gamma}\left(u_{0}+\sigma u_{1}\right)}{\partial t^{\gamma}}\right)\right|_{\sigma=0} \\
& =\frac{\partial^{\beta} u_{0}}{\partial y^{\beta}} \frac{\partial^{\gamma} u_{1}}{\partial t^{\gamma}}+\frac{\partial^{\beta} u_{1}}{\partial y^{\beta}} \frac{\partial^{\gamma} u_{0}}{\partial t^{\gamma}} \\
& =\left(y^{1-\beta} t\right)\left((2-\gamma) \frac{x^{2}}{2} y^{2-\beta} t^{2-2 \gamma}-\frac{x^{2}}{2} y t^{1-\gamma}\right)+\left((2-\beta) \frac{x^{2}}{2} y^{2-2 \beta} t^{2-\gamma}-\frac{x^{2}}{2} y^{1-\beta} t\right)\left(y t^{1-\gamma}\right) \\
& =\left((2-\gamma) \frac{x^{2}}{2} y^{3-2 \beta} t^{3-2 \gamma}-\frac{x^{2}}{2} y^{2-\beta} t^{2-\gamma}\right)+\left((2-\beta) \frac{x^{2}}{2} y^{3-2 \beta} t^{3-2 \gamma}-\frac{x^{2}}{2} y^{2-\beta} t^{2-\gamma}\right) .
\end{aligned}
$$

Therefore,

$$
\begin{aligned}
u_{2}=\mathrm{L}_{\mathrm{p}}^{-1} \mathrm{~L}_{\mathrm{q}}^{-1} \mathrm{~L}_{\mathrm{s}}^{-1} & \frac{1}{p^{2}} L_{x}^{\alpha} L_{y}^{\beta} L_{t}^{\gamma}\left(\left(\begin{array}{c}
\left((2-\gamma) \frac{x^{2}}{2} y^{3-2 \beta} t^{3-2 \gamma}-\frac{x^{2}}{2} y^{2-\beta} t^{2-\gamma}\right) \\
+\left((2-\beta) \frac{x^{2}}{2} y^{3-2 \beta} t^{3-2 \gamma}-\frac{x^{2}}{2} y^{2-\beta} t^{2-\gamma}\right) \\
-\left(\frac{x^{2}}{2} y^{2-\beta} t^{2-\gamma}-\frac{x^{2}}{2} y t+\frac{x^{3}}{3 !}\right)
\end{array}\right)\right. \\
- & \left(\begin{array}{c}
(2-\gamma) \frac{x^{4}}{4 !} y^{3-2 \beta} t^{3-2 \gamma} \\
-\frac{x^{4}}{4 !} y^{2-\beta} t^{2-\gamma}
\end{array}\right)+\left(\begin{array}{c}
(2-\beta) \frac{x^{4}}{4 !} y^{3-2 \beta} t^{3-2 \gamma} \\
-\frac{x^{4}}{4 !} y^{2-\beta} t^{2-\gamma} \\
=(4-\gamma-\beta) \frac{x^{4}}{4 !} y^{3-2 \beta} t^{3-2 \gamma}-\frac{x^{4}}{8} y^{2-\beta} t^{2-\gamma}+\frac{x^{4}}{4 !} y t-\frac{x^{5}}{5 !} .
\end{array}\right)-\left(\begin{array}{c}
\frac{x^{4}}{4 !} y^{2-\beta} t^{2-\gamma} \\
-\frac{x^{4}}{4 !} y t+\frac{x^{5}}{5 !}
\end{array}\right)
\end{aligned}
$$

The approximate series solution is,

$$
u(x, y, t)=(y t-x)+\left(\frac{x^{2}}{2} y^{2-\beta} t^{2-\gamma}-\frac{x^{2}}{2} y t+\frac{x^{3}}{3 !}\right)+\left(\begin{array}{c}
(4-\gamma-\beta) \frac{x^{4}}{4 !} y^{3-2 \beta} t^{3-2 \gamma} \\
-\frac{x^{4}}{8} y^{2-\beta} t^{2-\gamma}+\frac{x^{4}}{4 !} y t-\frac{x^{5}}{5 !}
\end{array}\right)+\ldots
$$




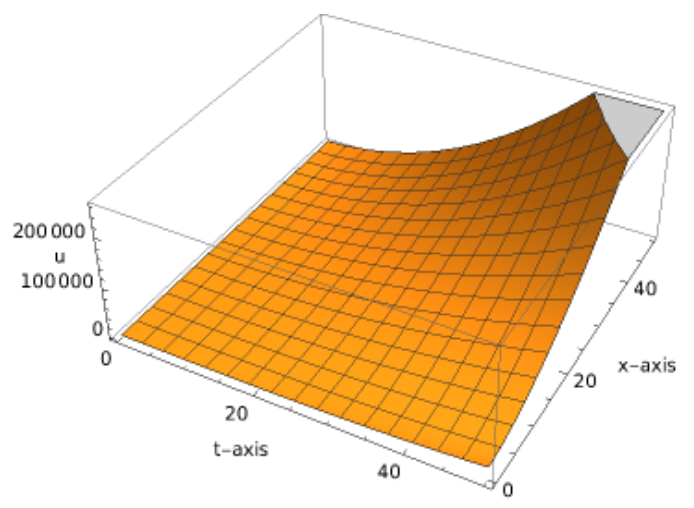

Figure 1: 3D Plot of Eq. (6.20) for $\gamma=0.50 ; \beta=0.25 ; y=1$.

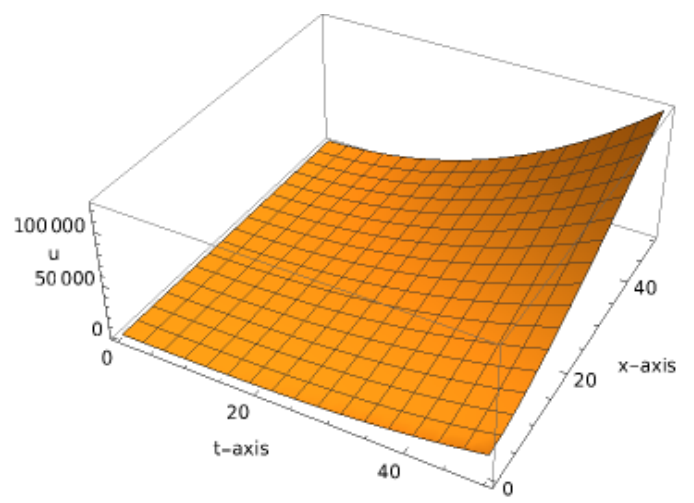

Figure 2: 3D Plot of Eq. (6.20) for $\gamma=0.75 ; \beta=0.50 ; y=1$.

for $\alpha, \beta, \gamma \in(0,1], x, y, t \in[0, \infty)$.

Note that for $\alpha, \beta, \gamma=1$, the solution of Eq. (6.11) from Eq. (6.20) as,

$$
u(x, y, t)=y t-\left(x-\frac{x^{3}}{3 !}+\frac{x^{5}}{5 !}+\ldots\right)=y t-\sin x .
$$

Figs. 1-4 show the 3D graphical representations for both the proposed methods, of Eq. (6.20) with various values of $\beta$ and $\gamma$.

\section{Conclusion}

In this work, the definition of fractional conformable triple Laplace and Sumudu transform are defined and investigated using all our obtained novel results and theorems with its systematic proofs. The new fractional conformable triple Laplace and Sumudu transform decomposition methods are given in the generalized form, to find the solution of 


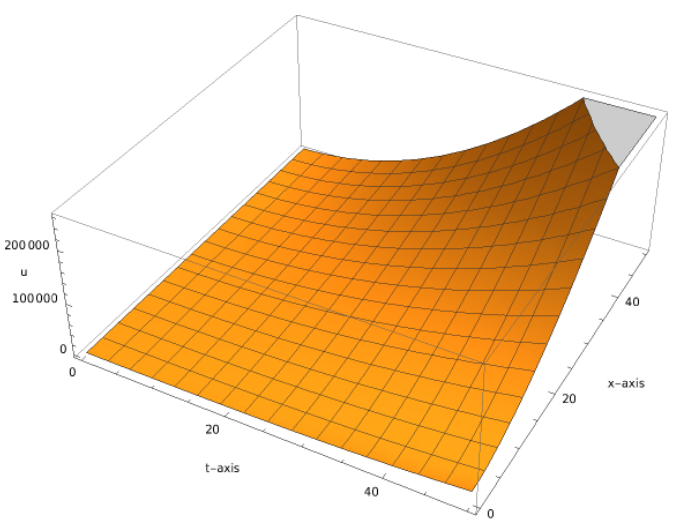

Figure 3: 3D Plot of Eq. (6.20) for $\gamma=0.50 ; \beta=0.50 ; y=1$.

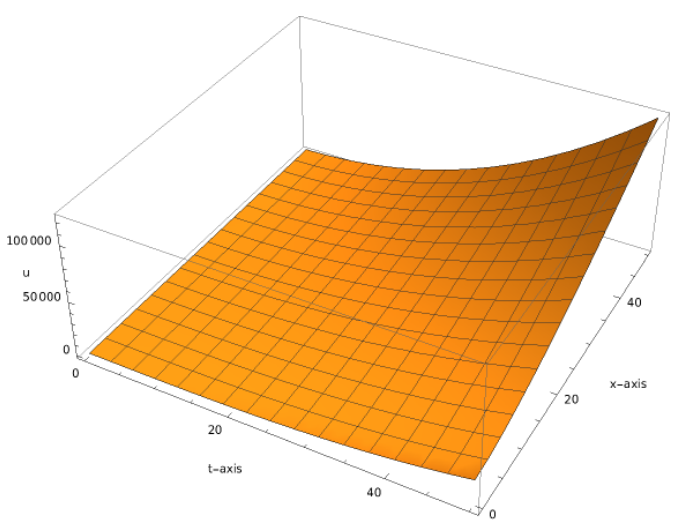

Figure 4: 3D Plot of Eq. (6.20) for $\gamma=0.75 ; \beta=0.75 ; y=1$.

nonlinear partial fractional differential equations. Finally, a numerical experiment has been conducted using the proposed methods - conformable Sumudu and Laplace decomposition method, and analyzed it. Noted the outcome of both methods: Laplace and Sumudu transforms decomposition method yields the same results and mentioned with the graphical representation as fig. 1,2,3,4. which leads to the answer of question raised in [31]. Infect, these methods can be led to solve simultaneous linear and nonlinear partial fractional differential equations.

\section{Acknowledgement}

We would like to thank the editors and the reviewers for their valuable comments on developing this paper and Dr Krunal B Kachhia, Faculty of Charusat University, Changa, Gujarat, India for his valuable suggestions and motivations for this research work: We also thank Prof Meghna Parikh, LJIET for their help developing the Tex file. 


\section{References}

[1] Kilbas A. A., Srivastava H. M. and Trujillo J. J., Theory and Applications of Fractional Differential Equations, Elsevier, Amsterdam, 2006.

[2] Podlubny I., Fractional Differential Equations, Academic Press, London, 1999.

[3] Avcı D., Iskender Eroglu B. B. and Ozdemir N., Conformable heat equation on a radial symmetric plate. Therm. Sci., 21 (2) (2017), 819-826.

[4] Khalil R., Horani M. A., Yousef A. and Sababheh M., A new definition of fractional derivative. J. Comput. Appl. Math., 26 (2014), 465-70.

[5] Yaslan H., New analytic solutions of the conformable spacetime fractional Kawahara equation. Optik, 140 (2017), 123-126.

[6] Korkmaz A., Hosseini K., Exact solutions of a nonlinear conformable time-fractional parabolic equation with exponential nonlinearity using reliable methods. Opt. Quantum Electron., 49 (8) (2017), 278.

[7] Hosseini K., Mayeli P. and Ansari R., Bright and singular soliton solutions of the conformable time-fractional Klein-Gordon equations with different nonlinearities. Waves Random Complex Media, 26 (2017), 1-9.

[8] Korkmaz A., On the wave solutions of conformable fractional evolution equations. Commun. Fac. Sci. Univ. Ank. Ser. A1, 67 (1) (2018), 68-79.

[9] Chen C., Jiang Y. L., Simplest equation method for some time-fractional partial differential equations with conformable derivative. Comput. Math. Appl., 75 (2018), 2978-2988.

[10] Hashemi M. S., Invariant subspaces admitted by fractional differential equations with conformable derivatives. Chaos Solitons Fractals, 107 (2018), 161-169.

[11] Özkan O., Kurt A., On conformable double Laplace transform. Optical and Quantum Electronics, 50 (2018), 1-9.

[12] Alfaqeih S., Kayijuka I., Solving system of conformable fractional differential equations by conformable double Laplace decomposition method. J. Part. Diff. Eq., 33 (2020), 275-290.

[13] Alfaqeih S., Misirli E., On double Shehu transform and its properties with applications. Int. J. Anal. Appl., 18 (2020), 381-395.

[14] Watugala G. K., Sumudu transform: a new integral transform to solve differential equations and control engineering problems. J. Math. Educ. Sci. Technol, 24 (1) (1993), 35-43.

[15] Asiru M. A., Further properties of the Sumudu transform and its applications. Int. J. Math. Educ. Sci. Technol., 33 (2) (2002), 441-449.

[16] Belgacem F. B. M., Introducing and analysing deeper Sumudu properties. Nonlinear Studies, 13 (1) (2006), 23-41.

[17] Tchuenche J. M., Mbare N. S., An application of the double Sumudu transform. Applied Math. Sci., 1 (2007), 31-39.

[18] Kilicman A., Eltayeb, H., On the applications of Laplace and Sumudu transforms. Journal of the Franklin Institute, 347 (5) (2010), 848-862.

[19] Al-Zhour Z., Alrawajeh F., Al-Mutairi N. and Alkhasawneh R., New results on the conformable fractional Sumudu transform: theories and applications. Int. J. Anal. Appl., 17 (6) (2019), 1019-1033.

[20] Abdeljawad T., On conformable fractional calculus. J. Comput. Appl. Math, 279 (2015), 57-66.

[21] Thabet H., Kendre S., Analytical solutions for conformable space time fractional partial differential equations via fractional differential transform. Chaos Solitons Fractals, 109 (2018), 238-245. 
[22] Ozkan O., Kurt A., Conformable fractional double Laplace transform and its applications to fractional partial integro-differential equations. J. Fract. Calc. Appl., 11 (1) (2020), 70-81.

[23] Mousa A., Elzaki T. M., Solution of nonlinear partial differential equations by triple Laplace decomposition method. International Journal of Engineering Applied Sciences and Technology, 4 (2019), 37-40.

[24] Alfaqeih, S., Bakcerler G. and Misirli E., Conformable double Sumudu transform with applications. Journal of Applied and Computational Mechanics, 7 (2021), 578-586.

[25] Al-Zhour Z., Alrawajeh F., Al-Mutairi N. and Alkhasawneh R., New results on the conformable fractional Sumudu transform: theories and applications. International Journal of Analysis and Applications, 17 (2019), 1019-1033.

[26] Gadain H. E., Solving coupled pseudo-parabolic equation using a modified double Laplace decomposition method. Acta Mathematica Scientia, 38 (2018), 333-346.

[27] Silva F. S., Moreira D. M. and Moret M. A., Conformable Laplace transform of fractional differential equations. Axioms, 7 (2018), 55-66.

[28] Kiliciman A., Omran M., On fractional double Laplace transform and its applications. J. Nonlinear Sci. Appl., 10 (2017), 1744-1754.

[29] Martínez F., Martínez I. and Paredes S., Conformable Euler s theorem on homogeneous functions. Comp. and Math. Methods, 1 (2019), e1048.

[30] Kaabar M., Novel methods for solving the conformable wave equation. Journal of New Theory, 31 (2020), 56-85.

[31] Atangana A., Akgl A., Can transfer function and Bode diagram be obtained from Sumudu transform. Alexandria Engineering Journal, 59 (4) (2020), 1971-1984.

[32] Eltayeb H., Mesloub S., Application of Conformable Sumudu Decomposition Method for Solving Conformable Fractional Coupled Burgers Equation. Journal of Function Spaces, 2021.

[33] Bulut Hasan, Haci Mehmet Baskonus and Fethi Bin Muhammad Belgacem. The analytical solution of some fractional ordinary differential equations by the Sumudu transform method. Abstract and Applied Analysis, Vol. 2013. Hindawi, 2013.

[34] Bhanotar Shailesh A., Kabbar Mohammed K. A., Analytical Solutions for the Nonlinear Partial Differential Equations using the Conformable Triple Laplace Transform Decomposition Method International Journal of Differential Equations, Vol. 2021. Hindawi (2021).

[35] Bhanotar Shailesh A., Belgacem F. B. M, On the Conformable Fractional Triple Sumudu Transform and its Applications. Lecture Notes in Electrical Engineering, Springer. (Accepted)

[36] Ibrahim R. W., Jahangiri J. M. and Center C. C., Conformable differential operator generalizes the Briot-Bouquet differential equation in a complex domain. AIMS Math., 4 (2019), 1582-1595.

[37] Ibrahim Rabha W., Rafida M. Elobaid and Suzan Obaiys J., Symmetric conformable fractional derivative of complex variables. Mathematics, 8 (2020), 363-375.

[38] Ibrahim R. W., Baleanu D., On quantum hybrid fractional conformable differential and integral operators in a complex domain. Revista de la Real Academia de Ciencias Exactas, Fsicas y Naturales. Serie A. Matemticas, 115 (1) (2021), 1-13.

[39] Ibrahim R. W., Elobaid R. M. and Obaiys S. J., On subclasses of analytic functions based on a quantum symmetric conformable differential operator with application. Advances in Difference Equations, 1 (2020), 1-14.

[40] Ibrahim Rabha W., et al. Analytic solutions of the generalized water wave dynamical equations based on time-space symmetric differential operator. Journal of Ocean Engineering and Science, 5 (2) (2020), 186-195. 
[41] Nashine H. K., Saluja G. S. and Ibrahim R. W., Some fixed point theorems for $(\psi-\phi)$-almost weak contractions in S-metric spaces solving conformable differential equations. Journal of Inequalities and Applications, 1 (2020), 1-27. 